\title{
ARTICLE
}

Received 19 Feb 2014 | Accepted 24 Dec 2014 | Published 28 Jan 2015 DOl: 10.1038/ncomms7175

\section{ADAM8 as a drug target in pancreatic cancer}

\author{
Uwe Schlomann ${ }^{1,2}$, Garrit Koller ${ }^{1}$, Catharina Conrad ${ }^{2}$, Taheera Ferdous' ${ }^{1}$ Panagiota Golfi ${ }^{1}$, Adolfo Molejon Garcia ${ }^{1}$, \\ Sabrina Höfling ${ }^{1}$, Maddy Parsons ${ }^{3}$, Patricia Costa ${ }^{4}$, Robin Soper ${ }^{4}$, Maud Bossard ${ }^{4}$, Thorsten Hagemann ${ }^{4}$, \\ Rozita Roshani ${ }^{4}$, Norbert Sewald ${ }^{5}$, Randal R. Ketchem ${ }^{6}$, Marcia L. Moss ${ }^{7}$, Fred H. Rasmussen ${ }^{7}$, Miles A. Miller ${ }^{8}$, \\ Douglas A. Lauffenburger ${ }^{8}$, David A. Tuveson ${ }^{9}$, Christopher Nimsky² \& Jörg W. Bartsch,2
}

Pancreatic ductal adenocarcinoma (PDAC) has a grim prognosis with $<5 \%$ survivors after 5 years. High expression levels of ADAM8, a metalloprotease disintegrin, are correlated with poor clinical outcome. We show that ADAM8 expression is associated with increased migration and invasiveness of PDAC cells caused by activation of ERK1/2 and higher MMP activities. For biological function, ADAM8 requires multimerization and associates with $\beta 1$ integrin on the cell surface. A peptidomimetic ADAM8 inhibitor, BK-1361, designed by structural modelling of the disintegrin domain, prevents ADAM8 multimerization. In PDAC cells, BK-1361 affects ADAM8 function leading to reduced invasiveness, and less ERK1/2 and MMP activation. BK-1361 application in mice decreased tumour burden and metastasis of implanted pancreatic tumour cells and provides improved metrics of clinical symptoms and survival in a $\mathrm{Kras}^{\mathrm{G} 12 \mathrm{D}}$-driven mouse model of PDAC. Thus, our data integrate ADAM 8 in pancreatic cancer signalling and validate ADAM8 as a target for PDAC therapy.

\footnotetext{
${ }^{1}$ King's College London, Institute for Pharmaceutical Science and KCLDI, London SE1 9RT, UK. ${ }^{2}$ Department of Neurosurgery, Marburg University, , Baldingerstrasse, 35033 Marburg, Germany. ${ }^{3}$ King's College London, Randall Institute, London SE1 8RT, UK. ${ }^{4}$ Institute of Cancer and Inflammation, St Mary's School of Medicine, John Vane Building, Charterhouse Square, London, EC1M 6BQ, UK. ${ }^{5}$ Department of Organic Chemistry, Bielefeld University, 33615 Bielefeld, Germany. ${ }^{6}$ Therapeutic Discovery, AMGEN Inc., Seattle, Washington 98119, USA. ${ }^{7}$ Biozyme Inc., Apex, North Carolina 27523, USA. ${ }^{8}$ Department of Biological Engineering, Massachusetts Institute of Technology, Cambridge, Massachusetts 02139, USA. ${ }^{9}$ Cold Spring Harbor Laboratory, Cold Spring Harbor, New York 11791, USA. Correspondence and requests for materials should be addressed to J.W.B. (email: jbartsch@med.uni-marburg.de).
} 
$\mathrm{P}$ ancreatic ductal adenocarcinoma (PDAC) has the highest mortality rate of solid organ cancers with a 5-year survival rate $<5 \%$ (ref. 1 ). This cancer type is remarkably homogenous in that $95 \%$ of PDAC originate from oncogenic mutations in the KRAS gene. Hence, representative authentic mouse models of PDAC with pancreas-specific expression of Kras have been generated ${ }^{2}$. In mice and man, KRAS mutations cause early-stage pancreatic intraepithelial neoplasias (PanINs) with subsequent development of progressive PDAC. A hallmark of PDAC is the massive infiltration of tumour cells into the pancreas and surrounding tissues including lymphatic organs, spleen and peritoneum, and the concomitant metastasis to the liver and lungs ${ }^{3-6}$. Infiltration of pancreatic tumour cells depends critically on extracellular matrix (ECM) remodelling ${ }^{7,8}$. Given the importance of the ECM in PDAC, the proteolytic release of membrane proteins (shedding) as well as ECM (e.g., collagens and fibronectin) degradation has previously been postulated to have a pivotal role in shaping the tumour microenvironment ${ }^{9,10}$. Members of the Metzincin superfamily, matrix metalloproteases (MMPs) and/or ADAM (A disintegrin and metalloproteinase) proteases have been described in these processes ${ }^{11}$. In particular, the contribution of ADAMs to extracellular remodelling ${ }^{12}$ and tumour growth, infiltration, metastasis and angiogenesis by shedding of membrane-associated proteins may be important ${ }^{9,13,14}$. In PDAC patient samples, elevated expression levels of ADAM8 (CD156a, MS2) have been identified versus normal pancreatic tissues. In normal pancreas, ADAM8 expression is very low and restricted to the plasma membrane of ductal cells and, to a lesser extent, of islets and acinar cells. In PDAC tissues, ADAM8 is strongly expressed in tubular complexes and in cancer cells. Based on clinical data, high ADAM8 expression levels are associated with a poor patient prognosis, resulting in reduced survival and increased metastatic spread $^{15}$.

ADAM8 is a proteolytically active member of the ADAM protease family originally described in inflammatory processes ${ }^{16-18}$ and subsequently in many systems of the body ${ }^{19}$. Increased expression of ADAM8 was observed in other neoplasias, such as high-grade glioma ${ }^{20}$, lung adenocarcinoma ${ }^{21}$, prostate cancer ${ }^{22}$ and, more recently, in squamous head and neck cell carcinoma ${ }^{23}$, medulloblastoma ${ }^{24}$, osteosarcoma ${ }^{25}$ and breast cancer ${ }^{26}$, suggesting that ADAM8 has an active role in tumour progression. Thus, understanding the functional role of ADAM8 in tumour biology is important.

ADAM8 is localized in a few distinct cell types and the analysis of ADAM8-deficient mice inferred dispensability for normal development and homeostasis ${ }^{27,28}$. ADAM8 is typically expressed at low levels, giving rise to the current hypothesis that it is functionally irrelevant for homeostasis unless induced by inflammatory stimuli ${ }^{17}$ or neoplasias. Once upregulated, ADAM8 can overlap with the substrate spectrum of ADAM10 and ADAM17, two major shedding enzymes, and cleave proteins with immune functions such as tumour necrosis factor receptor 1 (ref. 28), L-selectin ${ }^{29}$, CD23 (ref. 30) and CXCL1 (ref. 31), as well as cell adhesion proteins such as CHL1 (ref. 32), thereby potentially modulating immune response or cell adhesion. Cleavage of other ADAM8 substrates such as Tie-2, Flt-1, VE-cadherin, Flk-1, EphB4, KL-1, CD31 and E-selectin ${ }^{33}$ or by cleavage of fibronectin ${ }^{12}$ may control tumour angiogenesis. Moreover, a role for ADAM8 in metastases ${ }^{34}$ and in cell invasiveness ${ }^{15,20,22}$ has been postulated, although the mechanism underlying these processes is unknown.

ADAM8 is activated by autocatalysis in the trans-Golgi network $^{35}$ and, unlike other ADAMs, not by furine-like convertases. For in vivo activity, ADAM8 requires homophilic multimerization of at least two ADAM8 monomers on the cell membrane. This specific interaction of ADAM8 monomers offers a potential strategy for blocking ADAM8 activity by preventing ADAM8 multimerization in vivo. We have demonstrated previously that ADAM8-ADAM8 interactions critically depend on the disintegrin (DI)/cysteine (DC)-rich domain ${ }^{35}$.

In addition, ADAM family members can bind to integrins in vitro via their DC-rich domains ${ }^{36}$; as prototype, human ADAM15 contains a canonical RGD motif in the integrinbinding loop (IBL) of the DI domain ${ }^{37}$. However, even for nonRGD containing ADAMs such as ADAM9, integrin binding was demonstrated. ADAM9 binding to $\beta 1$ integrin causes migration of melanoma cells ${ }^{38}$, and for ADAM8, binding to $\alpha_{9} \beta_{1}$ was shown in osteoclast turnover ${ }^{39}$, suggesting that these ADAM-integrin interaction have functional relevance.

Although ADAM8 has been associated with increased tumour cell migration, invasiveness and metastasis via a combination of catalytic, adhesion and cell signalling functions ${ }^{15,20}$, no mechanistic data on ADAM8 in tumour progression and specifically in PDAC are available. Here we provide evidence for an involvement of ADAM8 in cancer signalling and in tumour progression. Furthermore, we validate ADAM8 as a target in PDAC by introducing a specific ADAM8 inhibitor.

\section{Results}

ADAM8 inhibition strategy. Cellular activation of ADAM8 occurs in two steps. The first is intracellular prodomain removal in vesicles, whereas the second is metalloprotease (MP) domain removal from membrane-bound-activated ADAM8 (Fig. 1a). Autocatalysis implies that ADAM8 multimerizes (Fig. 1a), and that the ADAM8 DC-rich domain is critical for multimerization as demonstrated previously by using an antibody directed against the DC domain 35 . To define the regions in the DC domain involved in ADAM8-ADAM8 interactions, homology modelling of the ADAM8 DC domain was performed based on the ADAM10 sequence derived from Janes et al. ${ }^{40}$ (Supplementary Figs 1 and 2). We hypothesized that an extended loop structure exposing the RGD-like positions of the 'KDX' motif in the IBL in human and mouse ADAM8 might be responsible for the observed interactions (Fig. 1b).

In mouse and human ADAM8, the amino-acid residues $\mathrm{K}$ and $\mathrm{D}$ are exposed towards the outer aspect of the DI domain, thereby forming a potential contact interface (Fig. 1b, labelled in magenta). To generate a peptidomimetic compound, a series of cyclic peptides (six amino acids) mimicking the motif 'RLSKDK' of mouse ADAM8 in the IBL were generated. Amino acids R, L or $\mathrm{S}$ were inserted in the peptide as D-amino acids to alter the conformational constraint of the KDK motif and to generate a potentially more stable peptide for in vivo work. The cyclic peptide sequence RLsKDK with 's' as D-serine named BK-1361 was most effective in blocking ADAM8-dependent cell adhesion in mouse and human cells with similar efficiencies (Supplementary Fig. 3) and ADAM8-ADAM8 interactions as shown by reduced FRET (Förster resonance energy transfer)/ FLIM (fluorescence lifetime imaging) efficiency. In contrast, a control peptide (CP; RLsADK; Fig. 1c) had no effect.

Multimerization of ADAM8 was investigated by native gel electrophoresis (Fig. 1d). Under native conditions, $100 \mathrm{ng}$ of proADAM8 associates in dimeric $(\sim 120 \mathrm{kDa})$ and trimeric $(\sim 180$ $\mathrm{kDa}$ ) complexes of pro-ADAM8, while in the presence of BK1361 , only monomers $(\sim 60 \mathrm{kDa})$ were detected (Fig. 1d, left panel). Detection of dimers and trimers suggests that dimers associate by DI domain (homophilic) interactions, whereas trimers could be formed by a different mode of interaction. At higher concentrations of recombinant ADAM8, we detected even larger complexes as a result of greater order multimerization 
a

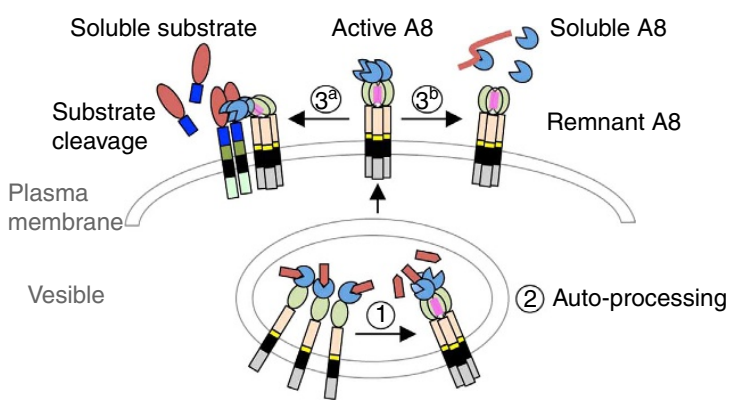

C

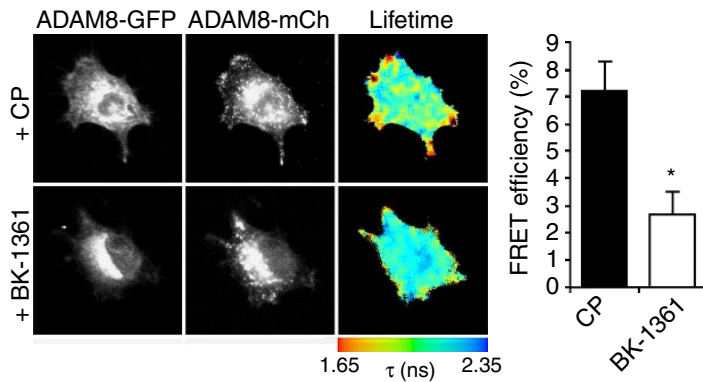

e

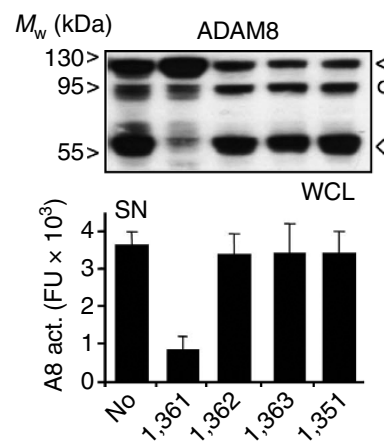

b

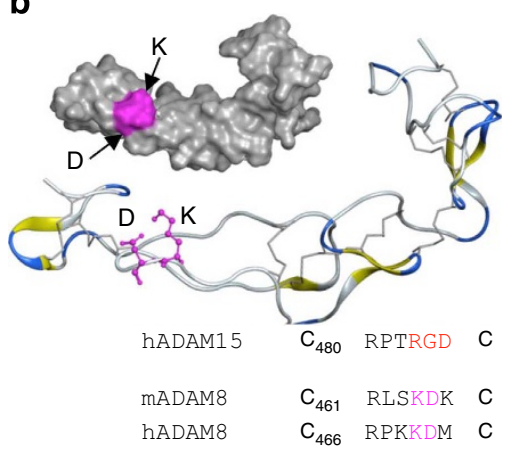

d

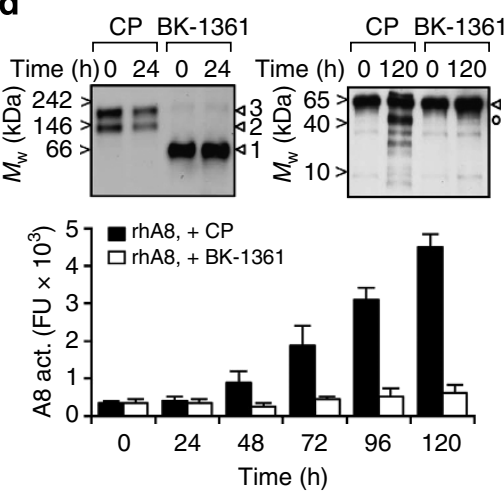

g
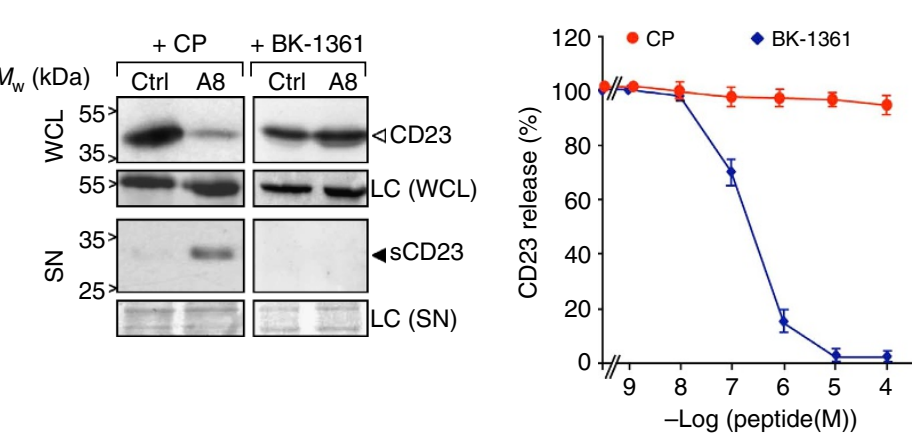

Figure 1 | Extracellular ADAM8 processing and inhibition of ADAM8 activity. (a) ADAM8 processing: (1) autocatalytic prodomain (red) removal for active ADAM8 (2); after membrane transport, ADAM8 cleaves membrane proteins (3a); alternatively, removal of a soluble MP (blue) domain (3b) leads to ECM cleavage and formation of remnant ADAM8. A putative interaction site (magenta) located in the disintegrin (DI) domain (green). (b) Homology modelling of ADAM8 DC-rich domain based on homology to pdb file 2ao7 (ref. 41) within the IBL region of ADAM15. The amino-acid motif 'KD' is potentially accessible to peptidomimetics such as BK-1361 (Table 1). (c) FRET analysis of ADAM8 monomers in the presence of BK-1362 (control peptide, $\mathrm{CP}$ ) and BK-1361 (500 nM) in Panc1 cells. FLIM (in nanoseconds, ns) and FRET efficiency are calculated from $>12$ cells in three independent experiments. ANOVA was used as statistical test; data are presented as mean \pm s.e.m.; ${ }^{*}<0.05$ (Student's $t$-test). (d) Left panel, complex of pro-ADAM8 (100 ng per lane) at 0 and $24 \mathrm{~h}$ analysed by native gel electrophoresis and immunoblotting. With CP, pro-ADAM 8 forms complexes (dimers and trimers, arrowheads 2 and 3); with BK-1361, only monomers of pro-ADMA8 are detected (arrowhead 1); right panel, activation of recombinant pro-ADAM8 in vitro in the presence of CP and BK-1361. Bar graph: Autocatalytic activation of pro-ADAM8 \pm BK-1361 (200 nM) in vitro. Pro-ADAM8 (100 ng) incubated for indicated times; Fluorescence activity using CD23 fluorogenic peptide monitored over 5 days in triplicates. FU, fluorescence units. (e) Effect of BK-1361 and CPs (Table 1) on ADAM8 MP domain removal in ADAM8-BiPro-transfected COS7 cells after $12 \mathrm{~h}$. Anti-BiPro antibody detects pro (triangle), mature (circle) and remnant (diamond) ADAM8 in cell lysates (WCLs); soluble ADAM8 activity from processed ADAM8 is detected in cell supernatants (SNs) by CD 23 peptide cleavage. (f) Test of BK-1361 for membrane-bound CD23 in WCLs and soluble CD 23 (sCD23) in SNs. Cells were incubated with CP or BK-1361 $(500 \mathrm{nM})$ for $12 \mathrm{~h}$; ctrl, control; LC, loading control. (g) Dose-dependent inhibition of CD23 shedding determined by ELISA $(n=5)$ with mean values $\pm \mathrm{s}$.d.; $\mathrm{IC}_{50}$ value for $\mathrm{BK}-1361$ was $182 \pm 23 \mathrm{nM}$, whereas $\mathrm{CP}$ shows no significant inhibition of CD23 shedding. ADAM8-mCherry, ADAM8-mCh.

(Supplementary Fig. 3e) in agreement with more than one interaction mode that results in ADAM8 complex formation. These interactions can be blocked by BK-1361, and we further analysed whether prevention of complex formation affects ADAM8 activity in vitro.
Activation of pro-ADAM8 was detected over a time course of $120 \mathrm{~h}$ (Fig. 1d, right panel) but blocked by BK-1361 (Fig. 1d, bar graph) with an $\mathrm{IC}_{50}$ of $120 \pm 19 \mathrm{nM}$. In a cell-based assay, BK1361 and other peptides (Table 1) were tested for their ability to inhibit ADAM8-dependent extracellular resulting in an active 


\begin{tabular}{|c|c|c|}
\hline Inhibitor & $\begin{array}{l}\text { A8 activation: } \\
\text { IC }_{50}(n M)\end{array}$ & $\begin{array}{l}\text { CD23 shedding: } \\
\text { IC }{ }_{50}(\mathrm{nM})\end{array}$ \\
\hline \multicolumn{3}{|l|}{ Hydroxamates } \\
\hline BB-94 (batimastat) & $46 \pm 4.5$ & $57 \pm 6$ \\
\hline BB-2516 (marimastat) & $1,054 \pm 154$ & $1,250 \pm 103$ \\
\hline \multicolumn{3}{|l|}{ Peptide inhibitors } \\
\hline cyclo-RLsKDK (BK-1361) & $120 \pm 19$ & $182 \pm 23$ \\
\hline cyclo-RISKDK (BK-1451) & $478 \pm 24$ & $570 \pm 23$ \\
\hline cyclo-rLSKDK (BK-2351) & $1,979 \pm 143$ & $2,430 \pm 87$ \\
\hline RLSKDK (BK-1351) & $>10,000$ & $>10,000$ \\
\hline cyclo-RLsADK (BK-1362) & $>5,000$ & $>10,000$ \\
\hline cyclo-RLsKAK (BK-1363) & $>5,000$ & $>5,000$ \\
\hline cyclo-RLsKDA (BK-1364) & $165 \pm 34$ & $255 \pm 27$ \\
\hline \multicolumn{3}{|c|}{$\begin{array}{l}\text { ADAM8, a disintegrin and metalloproteinase } 8 \text {. } \\
\text { IC } 50 \text { values of peptide variants tested for } A 8 \text { inhibition in vitro and for inhibition of } C D 23 \\
\text { shedding. Recombinant } A 8(100 \mathrm{ng} \text { total protein) was incubated for } 24 \mathrm{~h} \text { with the respective } \\
\text { inhibitors/peptides in a concentration of } 20-10,000 \mathrm{nM} \text {. A8 activity was monitored using the } \\
\text { CD23 fluorescence assay described in the Methods section. }\end{array}$} \\
\hline
\end{tabular}

soluble MP domain (Figs 1a and 3b). In cell lysates, presence of the remnant form indicates cellular processing of ADAM8 as seen with no or inactive CPs (Fig. 1e, upper panel). In cell supernatants (SNs), processing results in detectable activity of released ADAM8 MP. BK-1361, but not other BK peptide variants, decreased this activity (Fig. 1e, lower panel). BK-1361 blocks mouse and human ADAM8 in vitro with similar efficiencies, a prerequisite for testing BK-1361 in orthotopic PDAC models using human donor cells in mouse hosts.

We next tested BK-1361 and variants for their ability to inhibit shedding of CD23, a known substrate of ADAM8 (ref. 30) in cellbased shedding assays (Fig. If and Table 1). Co-transfection of ADAM8 with a tagged CD23 construct in COS7 cells resulted in significant shedding of $\mathrm{CD} 23$ as soluble $21 \mathrm{kDa}$ fragment (sCD23); sCD23 was detectable in SNs when ADAM8 was coexpressed. In the presence of $500 \mathrm{nM} \mathrm{BK}-1361$, sCD23 was undetectable, demonstrating that BK-1361 inhibits the in vivo shedding activity of ADAM8. Enzyme-linked immunosorbent assay (ELISA) assays were performed to determine [sCD23] versus [BK-1361] with an $\mathrm{IC}_{50}$ of $182 \pm 23 \mathrm{nM}$ for BK-1361; BK1362 had no significant effect (Fig. 1g). We conclude that BK1361 affects ADAM8-ADAM8 interactions, thereby inhibiting cellular shedding and autocatalytic activation of ADAM8 in vitro and in a cell-based assay in a specific manner, as neither catalytic activities of ADAM9, -10, -12 and -17 nor MMP-2, -9 and -14 were inhibited in concentrations of up to $10 \mu \mathrm{M}$ (Table 2).

ADAM8 induces migration/invasion of pancreatic cancer cells. As shown earlier, ADAM8 expression is correlated with invasiveness in vitro in various cell lines ${ }^{15,20,21}$. However, no functional in vivo data on ADAM8 in pancreatic malignancies are yet available. To establish cell lines for analysis of PDAC in vivo, we selected Pancl (very low ADAM8) and AsPC-1 cells (high endogenous levels of ADAM8) as determined by western blotting $^{15}$ (Fig. 2a). Pancl cell lines with a moderate overexpression of ADAM8 (Panc1_A8; NM_001109.4; Fig. 2b) and AsPC-1 cell lines with ADAM8 shRNA knockdown constructs were generated. Microarray analysis of Pancl_ctrl versus Panc1_A8 cells revealed that Panc1_A8 cells showed no off-target effects of the ADAM8 knockdown, as expression levels of $>95 \%$ of genes were unchanged, including genes encoding MMPs, other ADAMs, ADAMTS and TIMP1-4 levels
Table 2 | Specificity of BK-1361 against other ADAM proteases and MMPs.

\begin{tabular}{|c|c|c|}
\hline Protease & Peptide substrate & $\begin{array}{l}I C_{50} \\
(n M)\end{array}$ \\
\hline ADAM8 & $\begin{array}{l}\text { Dabcyl-His-Gly-Asp-GIn-Met-Ala-Gln-Lys-Ser- } \\
\text { Lys(Fam)-NH }{ }_{2}^{*}\end{array}$ & $120 \pm 19$ \\
\hline ADAM9 & $\begin{array}{l}\text { Dabcyl-Leu-Ala-Gln-Ala-Homophe-Arg-Ser- } \\
\text { Lys(Fam)-NH }{ }_{2}^{*}\end{array}$ & $>10,000$ \\
\hline ADAM10 & $\begin{array}{l}\text { Dabcyl-His-Gly-Asp-GIn-Met-Ala-Gln-Lys-Ser- } \\
\text { Lys(Fam)-NH }{ }_{2}^{*}\end{array}$ & $>10,000$ \\
\hline ADAM17 & $\begin{array}{l}\text { Dabcyl-Leu-Ala-Gln-Ala-Homophe-Arg-Ser- } \\
\text { Lys(Fam)-NH }{ }_{2}^{\star}\end{array}$ & $>10,000$ \\
\hline MMP-2 & Mca-PLGL-Dpa-AR-NH ${ }_{2}$ & $>10,000$ \\
\hline MMP-14 & Mca-KPLGL-Dpa-AR-NH ${ }_{2}$ & $>10,000$ \\
\hline
\end{tabular}

(Supplementary Fig. 4). Cellular localization of ADAM8, analysed by confocal laser-scanning microscopy, confirmed low ADAM8 expression in Panc1_ctrl cells compared with enhanced ADAM8 expression in Panc1_A8 cells. In Panc1_A8 cells, ADAM8 is localized in the cell membrane and membrane extrusions (Fig. 2b). In SNs of different Panc1_A8 cell clones characterized $(n=30)$, ADAM8 catalytic activity correlated with the $A D A M 8$ dosage, determined by western blotting (Fig. 2c) and CD23 fluorescence assay (Fig. 2d). Migration and invasion behaviour of Panc1_ctrl cells was compared with Panc1_A8 cells in vitro (Fig. 2e,f). In wound-healing (scratch) assays, migration rates of Panc1_A8 cells were significantly increased (8 \pm 3.8 -fold) compared with Panc1_ctrl cells. Panc1_A8 cell migration is inhibited by BB-94, a $\overline{M P}$ inhibitor. In addition, application of BK-1361 reduced migration rates of Panc1_A8 cells significantly, but not to the same level as BB-94, suggesting that ADAM8 modulates other MP activities accounting for migration above control levels. Accordingly, invasion into different ECM substrates such as collagen I, collagen IV, fibronectin and Matrigel was enhanced by ADAM8 (Fig. 2f).

ADAM8 increases extracellular MMP activity. A proteolytic activity matrix assay (PrAMA) ${ }^{41}$ was used for simultaneous detection of multiple activities in Panc1_ctrl and Panc1_A8 cells that could account for the observed invasiveness. Briefly, PrAMA is based on the knowledge of individual FRET-substrate MMP/ ADAM cleavage signatures using purified enzymes ${ }^{41}$. Panels of FRET-substrate cleavage measurements can be used to infer a dynamic, quantitative and specific profile of MMP/ADAM proteolytic activities from complex enzyme mixtures such a SNs and solubilized membranes (see Methods and Supplementary Fig. 5). PrAMA inference revealed increased activities of MMP-2 in SNs and MMP-14 (MT1-MMP) in cell membranes of Panc1_A8 cells compared with Panc1_ctrl cells (Fig. 2g and Supplementary Fig. 5). Enhanced ADAM8 activities were detected in SNs and membranes of Panc1_A8 cells. Gelatine zymography and western blotting for MMP-2 and MMP-14 confirmed increased activity of MMP-2 and higher membrane concentration of MT1-MMP (Fig. 2h). However, elevated MMP activities in Panc1_A8 cells are not due to transcriptional activation of $M M P-2$ and $M M P-14$ (Supplementary Fig. 4).

We further investigated whether BK-1361 is able to affect ADAM8-dependent invasiveness and MMP secretion of Panc1_A8 cells (Fig. 2i,j). BK-1361 and peptide variants were tested for their ability to block invasiveness of Panc1_A8 cells (Fig. 2i). A dose-dependent effect of BK-1361 on invasion of 
a

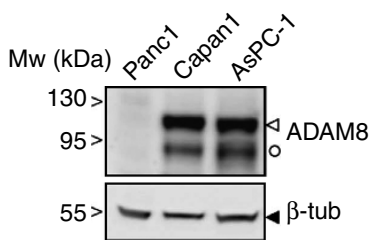

e

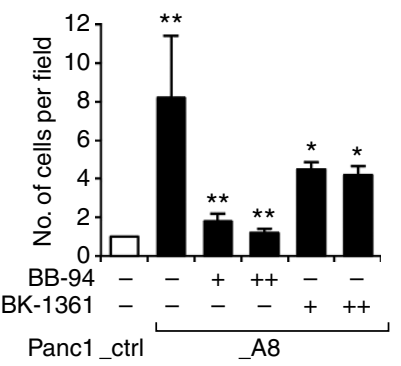

b

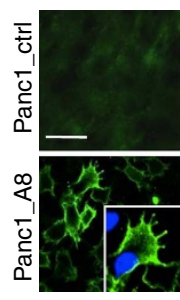

C

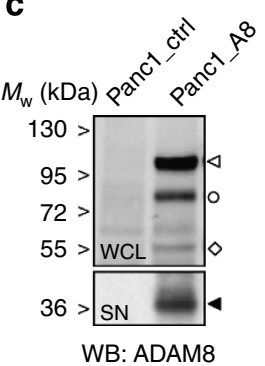

d
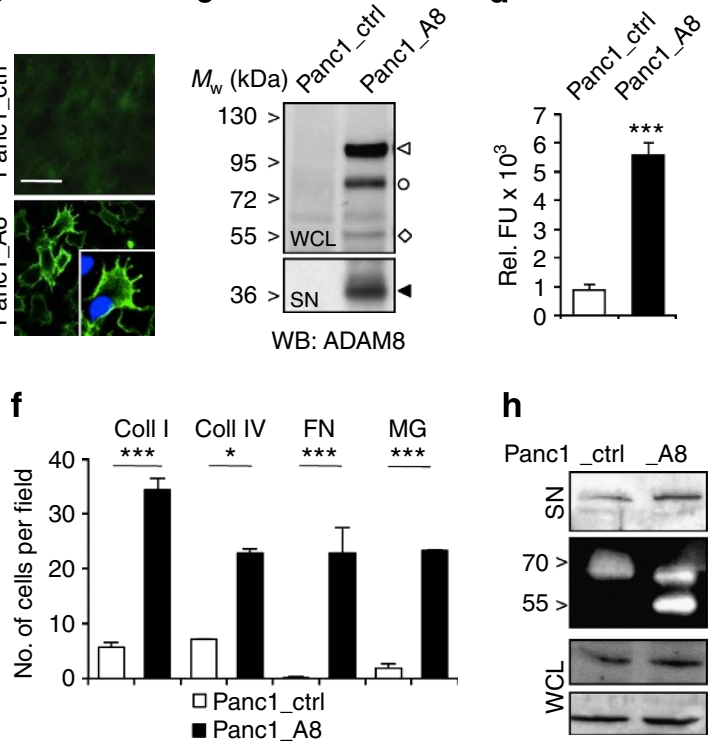

h

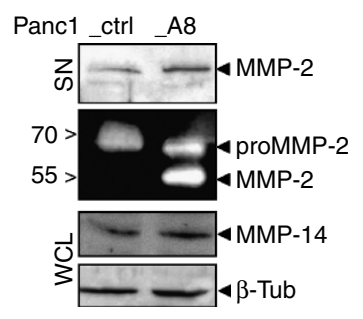

g

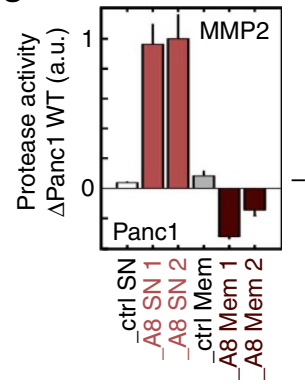

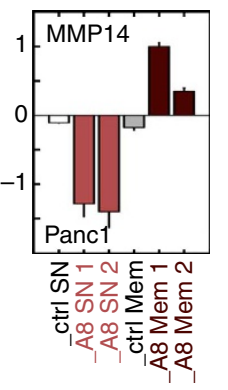

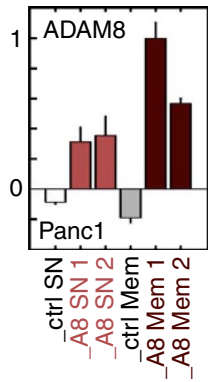

i

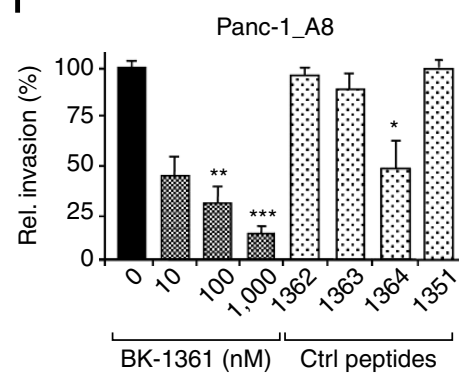

j

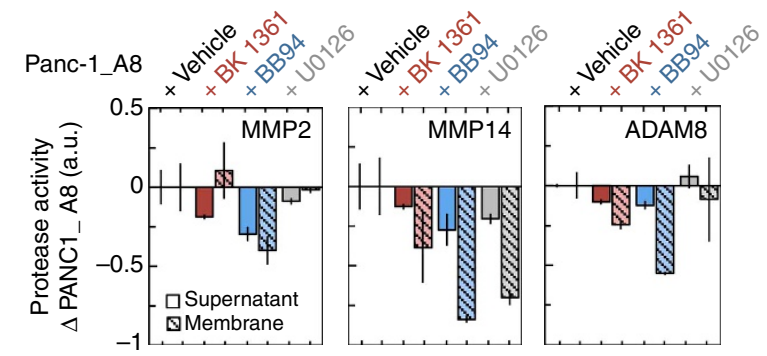

Figure 2 | Effect of ADAM8 expression in Panc1 cells. (a) Western blotting (WB) of ADAM8 in PDAC cell lines Panc1, Capan-1 and AsPC-1. (b) ADAM8 immunofluorescence (anti-ADAM8, green) in non-permeabilized Panc1_ctrl and Panc1_A8 cells. ADAM8 membrane staining in Panc1_ctrl (upper panel) and Panc1_A8 (lower panel) cells. Insert (lower panel), ADAM8 localization in membrane protrusions. Scale bar, $50 \mu \mathrm{m}$. (c) WB of whole-cell lysates (WCLs) and supernatants (SNs) from Panc1_ctrl and Panc1_A8 cells; proform (arrowhead), mature (circle) and remnant form (diamond) of ADAM8, respectively. In Panc1_A8 SN, an 30-kDa soluble ADAM8 MP fragment is detectable (filled arrowhead). (d) ADAM8 activities in SNs of Panc1_ctrl and Panc1_A8 cells were measured as fluorescence units (FU; $n=5$ ) by cleavage of CD23 substrate; ${ }^{\star \star \star} P<0.0001$ (Student's $t$-test); rel, relative. (e) Scratch assay of Panc1_ctrl versus Panc1_A8 cells treated either with BB-94 (100 nM '+' and 1 $\left.\mu M^{\prime}+++^{\prime}\right)$ or with BK-1361 (200 nM '+ ' and $500 \mathrm{nM}$ '+ + '). Cell counts after $t=0$ and $12 \mathrm{~h}$ (three independent experiments in triplicates, $n=9$ ); ANOVA was used as statistical test; ${ }^{\star} P<0.01,{ }^{\star \star} P<0.001$ (Student's t-test). (f) Invasion of Panc1_ctrl and Panc1_A8 cells in collagen I (Coll I), collagen IV (Coll IV), fibronectin (FN) and Matrigel (MG) analysed in Boyden chambers (18 h). Data are presented as mean \pm s.d. from five independent experiments. ANOVA test was used. ${ }^{\star} P<0.01,{ }^{\star \star \star} P<0.0001$ (Student's $t$-test). (g) PrAMA inference analysis of SNs and membranes (Mem) from Panc1_ctrl and Panc1_A8 cells using five fluorogenic peptides to detect MMP activities. Activities of MMP-2, MMP-14 and ADAM8 are shown as relative changes to Panc1_ctrl. (h) WB and gelatine zymography of Panc1_ctrl and Panc1_A8 SNs for MMP-2, MMP-2 activity and MMP-14. (i) Invasion of Panc1_A8 cells treated with BK-1361 (10, 100 and 1,000 nM) or 1,000 nM CPs for $6 \mathrm{~h}$ (Table 1). Invasion was determined after $18 \mathrm{~h}$. ANOVA test was used. ${ }^{\star} P<0.005,{ }^{\star \star} P<0.001,{ }^{\star \star \star} P<0.0001$. (j) PrAMA from Panc1_A8 cells treated with vehicle, BK-1361, BB-94 and U0126 (1 $\mu \mathrm{M}$, each) for $24 \mathrm{~h}$. Values are given relative to Panc1_A8 treated with vehicle only. ANOVA was used as statistical test. 
Panc1_A8 cells was observed. From CPs, only BK-1364 had a slight effect on invasion. In parallel, we performed PrAMA assays in Panc1_A8 cells to evaluate MMP-2, MMP-14, and ADAM8 activities in the presence of BK-1361, BB-94 and the extracellular regulated kinase (ERK) inhibitor U0126, respectively (Fig. 2j). BK-1361 and BB-94 reduced activities of MMP-2, MMP-14 and ADAM8. ERK inhibition had a slight effect on MMP-2 activity and a greater effect on MMP-14 activity, whereas ADAM8 activity was not affected. These findings argue for an ERK1/2mediated effect on MMP-14, and, to a lesser extent, on MMP-2 activation. ADAM8 as regulator of ERK1/2 activation is not directly affected by U0126.

Our data suggest an effect of pharmacological ADAM8 inhibition on invasiveness of PDAC cells. To determine the effect of a genetic ADAM8 knockdown on cellular invasiveness, we selected AsPC-1 cells with high endogenous ADAM8 levels (Fig. 2a) and generated AsPC_1 cell clones carrying a stable knockdown of ADAM8 (sh_A8). Three representative cell clones from different sh_A8 constructs were analysed for ADAM8 expression (Fig. 3a-c), cell migration and invasion (Fig. 3d,e and Supplementary Movies 1, 2). Knockdown of ADAM8 in AsPC-1 cells caused a significant drop in cell migration depending on the gene dosage of $A D A M 8$. Invasion of AsPC-1 cells was similarly affected by $A D A M 8$ dosage (Fig. 3e). BK-1361 treatment of wildtype (wt) AsPC-1 cells was similar to the genetic knockdown of ADAM8 with $87 \pm 3.5 \%$ inhibition (Fig. 3f). PrAMA assays were performed with AsPC-1_shCtrl \pm BK-1361 and AsPC-1_shA8 cell clones 1 and 2 (Fig. 3g). Reduction of MMP-2 and MMP-14 activities were observed in AsPC-1_shA8 clones. In BK-1361treated AsPC-1_shCtrl cells, MMP-2 was similarly affected, however, the effect on MMP-14 was less pronounced (Fig. 3g).

ADAM8 interacts with integrin $\beta 1$ in pancreatic cancer cells. The membrane localization of ADAM8 in Panc1_A8 cells suggests that ADAM8 is complexed with cellular integrins, thereby enhancing cell migration and invasiveness. To investigate this, co-immunoprecipitation (co-IP) experiments were performed in Panc1 cells expressing either control or a tagged ADAM8 construct (ADAM8-BiPro). As a result, $\beta 1$ integrin, present in comparable amounts in Panc1_ctrl and Panc1_A8 cells, was co-IP with ADAM8 (Fig. $4 \mathrm{a}-\mathrm{c}$ ). To analyse cellular ADAM8- $\beta 1$ a

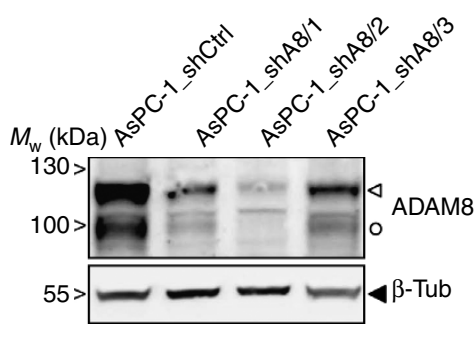

b

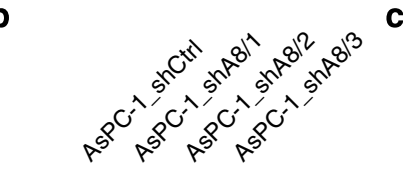

C
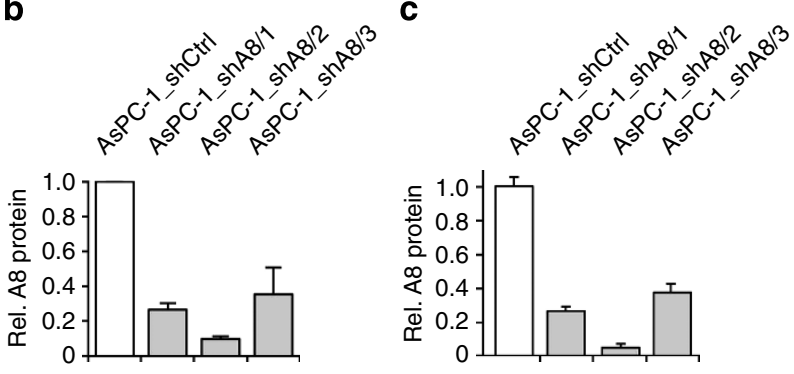

d

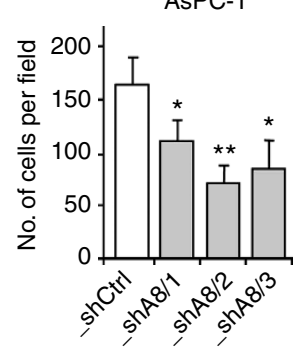

AsPC-1

$\mathbf{f}$
AsPC-1

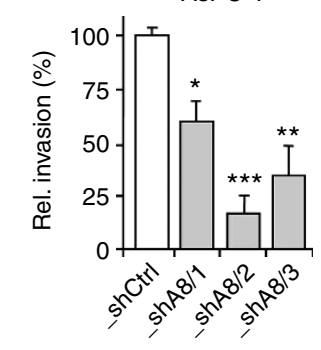

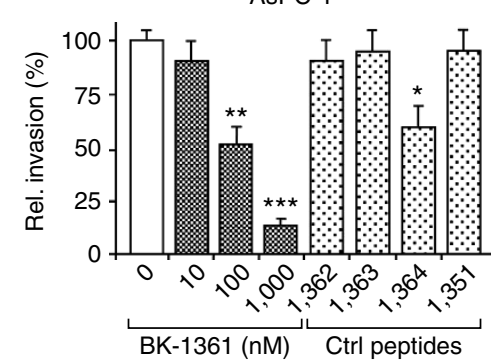

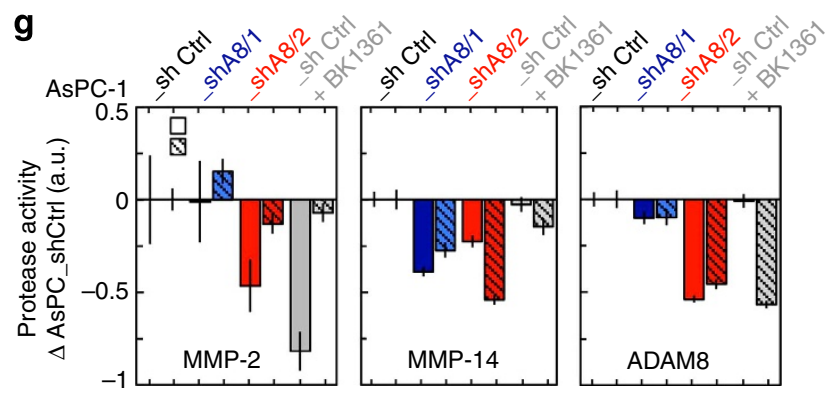

Figure 3 | ADAM8 inhibition reduces MMP-2 and MMP-14 activity and affects invasiveness. (a) Stable ADAM8 knockdown (shA8) of AsPC-1 cell clones were generated and characterized for ADAM8 protein (b) and mRNA (c) expression; rel, relative. Values are given as mean values from three independent experiments performed in triplicates \pm s.e.m. Using these cell clones, (d) scratch and (e) Matrigel invasion assays were performed. In both assays, an ADAM8 gene dosage-dependent effect was observed. (f) Matrigel invasion was determined after addition of BK-1361 (0-1,000 nM) or CPs (Table 1). For $\mathbf{d}-\mathbf{f}$, values are given as mean values \pm s.e.m. ANOVA was used as statistical test. ${ }^{\star} P<0.1,{ }^{\star \star} P<0.01$, ${ }^{\star \star \star} P<0.005$ (Student's $t$-test). (g) PrAMA inference analysis of two AsPC-1 ADAM8 knockdown clones and of AsPC-1_shCtrl cells treated with BK-1361, relative to AsPC-1_shCtrl cells. Note that both genetic and pharmacological inhibition of ADAM8 show similar effects, that is, reduction of MMP-2 and MMP-14 activity with the exception of MMP-14 in AsPC-1 cells. 
a

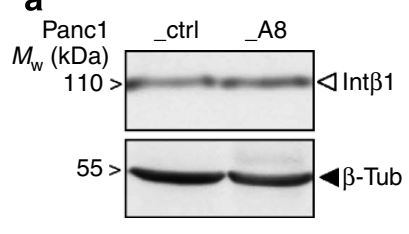

b

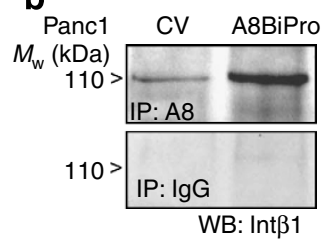

C

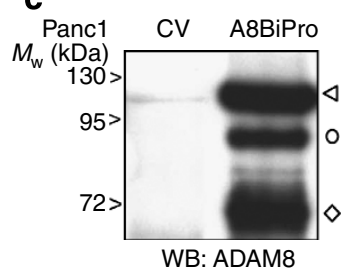

h
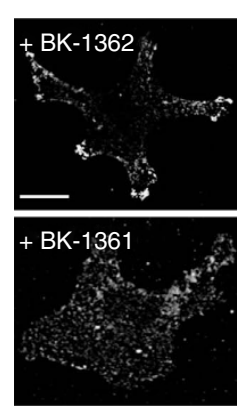

$\mathbf{k}$

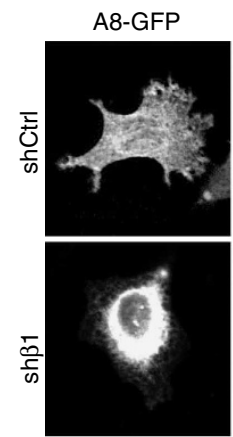

d
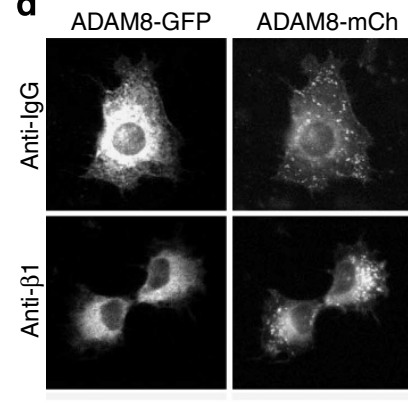

$\mathbf{f}$

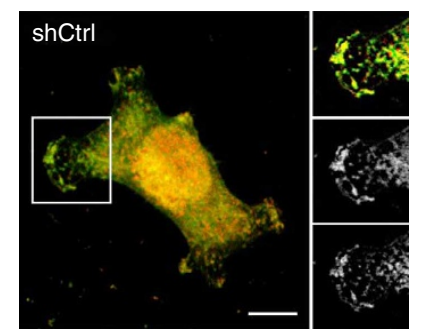

i

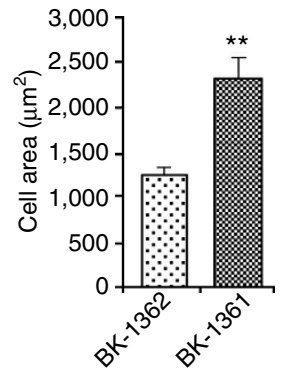

e

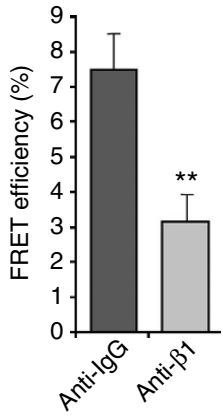

g

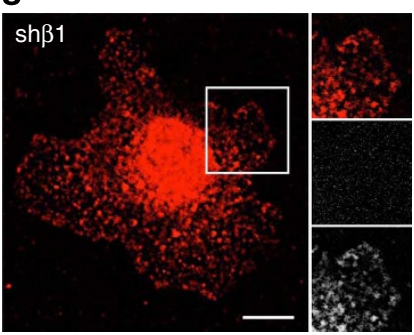

j

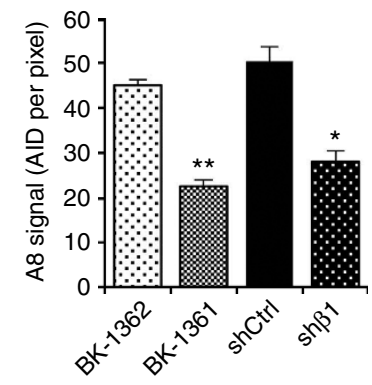

I
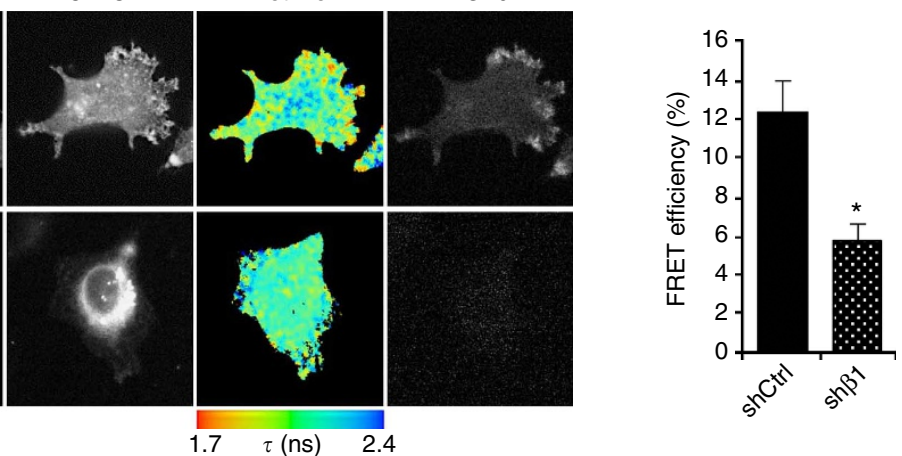

Figure 4 | ADAM8 multimerizes and interacts with integrin $\boldsymbol{\beta 1}$ in tumour cells. (a) Western blot (WB) of integrin $\beta 1$ subunit (Int $\beta 1$ ) in Panc1_ctrl (ctrl) and Panc1_A8 (A8) cells ( $\sim 15 \mathrm{kDa})$. $\beta$-Tubulin ( $\beta$-tub) was used as loading control. (b) In Panc1 cells transiently expressing control vector (CV) or ADAM8-Bipro (A8BiPro), anti-BiPro or unrelated lgG was used for immunoprecipitation (IP). Blots were probed with polyclonal $\beta 1$ integrin antibody. (c) Reprobing of blot (b) using polyclonal anti-ADAM8 antibody. (d) FRET/FLIM analysis of ADAM8-ADAM8 interactions. Panc1 cells were co-transfected with ADAM8-GFP and ADAM8-mCherry (A8-mCh) constructs; (upper panel) FLIM in the absence (control lgG) and (lower panel) in the presence of anti- $\beta 1$ antibody (anti- $\beta 1$ ), determined in nanoseconds (ns). (e) FRET efficiency (in \%) for ADAM8-ADAM8 interaction in anti- $\beta 1$ antibody-treated Panc1 cells $(n=10)$. As statistical test, ANOVA was used; ${ }^{\star \star} P<0.001$. (f-h) Localization of ADAM8 protein in MDA-MB-231 control (shCtrl, f) or (h) in MDA-MB-231 cells with a stable $\beta 1$ integrin knockdown. Staining of ADAM8 in red and $\beta 1$ integrin in green. Boxed areas in $\mathbf{f}$ are a lamellipod structure zoomed in. In contrast to boxed area in $\mathbf{f}$, the one in $\mathbf{g}$ shows diffuse staining of ADAM8 mainly in vesicles, the entire cell is flattened and cell area increases significantly.

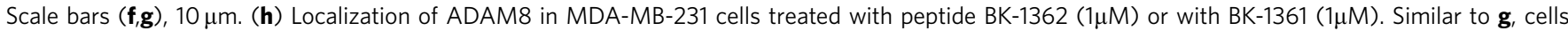
flatten and localization of ADAM8 in lamellipodia is changed. (i) Quantification of cell areas of BK-1362- and BK-1361-treated cells, given as mean \pm s.d. from 50 cell areas. ${ }^{\star \star} P<0.001$ (Student's $t$-test). (j) Quantification of ADAM8 in the cell membrane as average integrated density (AID) per pixel (sum of density $5 \mu \mathrm{m}$ in from cell periphery, divided by total pixels). ANOVA was used as statistical test. ${ }^{\star} P<0.01,{ }^{\star *} P<0.001$ (Student's $t$-test). (k) Interaction of ADAM8-GFP and ADAM8-mCh in MDA-MB-231 control (shCtrl) and MDA-MB-231 with a stable $\beta 1$ integrin knockdown (sh $\beta 1$ ). In the right panel, antibody 12 G10 was used to detect activated $\beta 1$ integrin. (I) Quantification of FRET efficiency between ADAM8-GFP and ADAM8-mCh in MDA-MB-231 control (shCtrl) and MDA-MB-231 with stable $\beta 1$ integrin knockdown (sh $\beta 1$ ). ANOVA was used; ${ }^{\star} P<0.01$ and ${ }^{\star \star} P<0.001$ (Student's $t$-test). 
integrin and ADAM8-ADAM8 interactions, FRET/FLIM analyses were performed to detect FRET in cell lines expressing fusion proteins ADAM8-GFP and ADAM8-mCherry, respectively. ADAM8 multimerization was detected in Pancl cells (Fig. $4 \mathrm{~d}, \mathrm{e}$ ), indicated by FRET efficiency of $7.5 \pm 0.93 \%$. In cells, complex formation and membrane localization of ADAM8 in vivo involves $\beta 1$ integrin, since treatment of Pancl cells with a $\beta 1$ integrin-blocking antibody (Fig. $4 \mathrm{~d}, \mathrm{e})$ resulted in a significant drop to $3.1 \pm 0.8 \%$ FRET efficiency. Moreover, MDA-MB-231 breast cancer cells lacking $\beta 1$ integrin (Fig. $4 \mathrm{f}, \mathrm{g}$ ) show a significant change in cellular morphology while ADAM8 localization in lamellipod structures is lost. Interestingly, administration of BK1361 causes a similar change in cell morphology (Fig. 4h,i). We conclude that $\beta 1$ integrin knockdown or specific ADAM8 inhibition have similar effects on ADAM8 membrane localization (Fig. 4j). In addition, areas of positive ADAM8-FRET in MDA-MB231 cells were analysed for $\beta 1$ integrin (Fig. 4k,l). In most areas, activated $\beta 1$ integrin was detected by antibody $12 \mathrm{G} 10$, suggesting that ADAM8 interaction and $\beta 1$ integrin activation are correlated.

To analyse whether ADAM8 interactions cause altered intracellular signalling, a mitogen-activated protein kinase (MAPK) array was used to screen for kinase phosphorylation in Panc1_ctrl and Panc1_A8 cells (Supplementary Fig. 6). As potential downstream effectors of the observed ADAM8- $\beta 1$ integrin interaction, we investigated phosphorylation of focal adhesion kinase (FAK), ERK1/2, Akt and p38 $\gamma$ in Panc1_A8 versus Panc1_ctrl cells. Focal adhesion kinase was described as $\beta 1$ integrin interacting protein ${ }^{42}$. In western blots, increased phosphorylation of FAK at residue Tyr397 correlates with ADAM8 expression levels in Pancl cells (Fig. 5a). In addition to pFAK, we detected increased phosphorylation of ERK1/2 (p44/ p42) in Panc1_A8 cells by western blotting using corresponding phospho-specific antibodies (3.4-fold \pm 0.2 Panc1_A8 versus Panc1_ctrl; Fig. 5b). The observed increase in pERK1/2 was reduced in Panc1_A8 cells treated with BK-1361 (Fig. 5b). To correlate ERK1/2 phosphorylation with the observed invasiveness of Pancl cells, Matrigel invasion assays were performed in the presence of U0126 (Fig. 5c). U0126 blocked ERK1/2 phosphorylation in Panc1_ctrl and Panc1_A8 cells, and resulted in decreased invasion of Panc1_A8 cells. In addition, AsPC1_shCtrl and three AsPC-1_shA 8 cell clones with different ADAM8 levels were analysed for pERK1/2 levels (Fig. 5d). In cell clone AsPC-1_shA8/2, pERK1/2 levels were reduced by $2.9 \pm 0.3$ fold, suggesting that ADAM8 expression levels are correlated with pERK1/2. In AsPC-1 cells, ADAM8 levels affect MEK1/2, p-Akt and $\mathrm{c}$-Raf activation (Fig. 5e). In addition, a $\beta 1$ integrin antibody that blocks activation was able to reduce pERK1/2 levels in Panc1_A8 cells, demonstrating that $\beta 1$ integrin is required for ADAM8-dependent ERK1/2 activation (Supplementary Fig. 7).

To investigate whether the observed FAK and ERK1/2 activation depends on membrane-bound ADAM8, Pancl cells were transfected with wt ADAM8 (A8 wt), an ADAM8 construct lacking the cytoplasmic domain $(\mathrm{A} 8 \Delta \mathrm{CD})$, or a soluble ADAM8 (A8ecto) construct (Fig. 5f). First, we confirmed that all constructs are catalytically active, as all three ADAM8 proteins shed CD23 (sCD23) from the cell membrane. Interestingly, neither $\Delta C D$ nor the ectodomain of ADAM8 were able to activate FAK and ERK1/2 (Fig. 5f), suggesting that intracellular signalling mediated by ADAM8 requires membrane localization of ADAM8 and the presence of the cytoplasmic domain. As potential substrates for ERK activation, that is, the epidermal growth factor receptor (EGFR) ligand family such as Heparin-binding epidermal growth factor (HB-EGF), EGF or amphiregulin, were screened (Supplementary Fig. 8). We have not identified significant EGFR ligand release, so that ADAM8- $\beta 1$ integrin interactions might act independent from EGFR signalling.
Role of ADAM8 in PDAC and effect of BK-1361 in vivo. Based on our findings that ADAM8 inhibition blocks invasiveness and ERK1/2 signalling in PDAC cells, we used BK-1361 to validate ADAM8 as a therapeutic target in PDAC in vivo. Initially, acute and chronic toxicity analyses were performed in C57BL/6J mice. In single and repeated dose applications, doses of up to $10 \mu \mathrm{gg}^{-1}$ body weight were well tolerated as mice showed no abnormalities, weight loss or motor performance over 4 weeks. After necropsy, organs investigated showed no abnormalities at histological level (Supplementary Fig. 9). Owing to the lack of acute and chronic toxicity, a dose of $10 \mu \mathrm{gg}^{-1}$ body weight was applied daily for subsequent in vivo applications.

Initially, orthotopic injections of Panc1 cells into mouse pancreas were performed (Fig. $6 \mathrm{a}-\mathrm{g})$ in three cohorts $(n=12$ each); cohort 1 received Panc1_A8 cells, cohort 2 received Panc1_ctrl cells and cohort 3 received Panc1_A8 cells followed by daily intraperitoneal injection of $10 \mu \mathrm{gg}^{-1}$ BK-1361. Mice were monitored for 12 days, by which time most of the mice injected with Panc1_A8 cells were moribund and reached end point criteria. In contrast, mice injected with Panc1_ctrl cells or Panc1_A8/BK-1361 treatment showed improved clinical parameters. At end point, pancreatic tumours formed from Panc1_ctrl cells were significantly smaller than from Panc1_A8 cells (Fig. 6a,b). Moreover, tumours obtained from mice that received Panc1_A8 cells and daily injections of BK-1361 were significantly smaller. These data indicate that inhibition of ADAM8 reduced tumour load to almost the value of Pancl_ctrl-derived tumours (0.42 g for Panc1_ctrl versus $0.62 \mathrm{~g}$ for Panc1_A8/BK-1361). By histology, a significant invasion of Panc1_A8 cells into the pancreatic tissue was detected, whereas in tumours derived from Panc1_A8/BK-1361 cells, tumour masses embedded in Matrigel were primarily localized to the implantation site even after 12 days, as the boundaries of pancreas and implanted tumour mass were still distinct (Fig. 6c). In addition, there were signs of necrosis inside the implanted tumour treated with BK-1361 (Fig. 6c), inferring that non-invasive Pancl cells undergo necrotic changes. Moreover, ADAM8 levels in Panc1_ctrl cells located in the tumour were increased under hypoxic conditions. Co-staining for pERK1/2 was observed in infiltrative ADAM8-positive tumour cells (Fig. 6d, upper panel).

Metastasis and infiltration is the major cause for the observed morbidity in PDAC 5 ,6. Since ADAM8 was discussed in the context of infiltration and metastasis ${ }^{26,34}$, we investigated orthotopic mice for infiltration of close structures such as peritoneum, diaphragm and spleen, and liver metastasis. From mice injected with Panc1_A8 cells, we found significant infiltrates in adjacent organs (Fig. 6e,f). Macroscopic inspection and haematoxylin/eosin stain of tissue sections revealed higher invasion into spleen and diaphragm of Panc1_A8injected mice (Fig. 6e). Analysis of infiltration areas showed enhanced invasive behaviour of Panc1_A8 cells versus Panc1_ctrl and Panc1_A8/BK-1361 cells (Fig. 6f) in peritoneum, diaphragm and spleen.

ADAM8 staining of liver sections revealed occurrence of micrometastases with higher frequencies in Panc1_A8 implanted mice compared with Panc1_ctrl and Panc1_A8/BK-1361 (Fig. 6g). Metastases frequencies were markedly different between Panc1_A8, Panc1_ctrl and Panc1_A8/BK-1361. Furthermore, the implantation of AsPC-1_shCtrl and AsPC-1_shA8 cells was analysed (Fig. 6h). AsPC-1_shCtrl cells caused large streams of tumour cells invading the pancreatic tissue with an infiltration area of $21 \pm 2.8 \%$. In contrast, AsPC-1_shA8 cells were located close to the injection site and showed less invasive behaviour with infiltration areas of $3.4 \pm 1.2 \%(P<0.01)$. Thus, data derived from genetic ADAM8 knockdown support the results obtained with ADAM8 inhibition using BK-1361. 
a

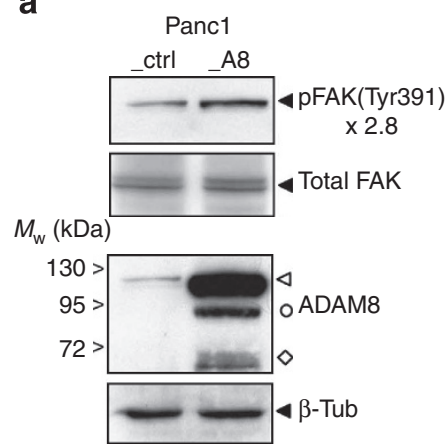

d

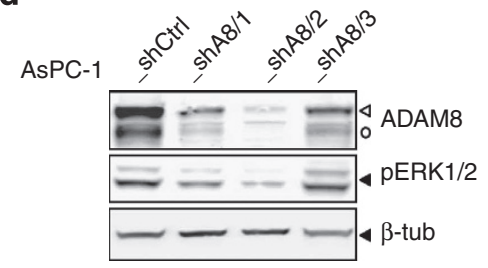

$e_{\text {AsPC-1 }}$

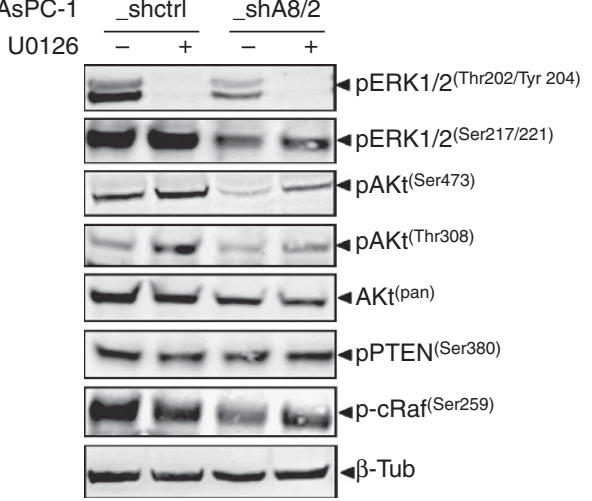

b

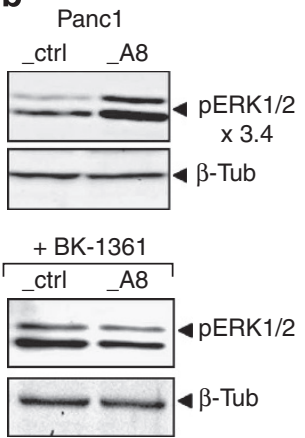

C

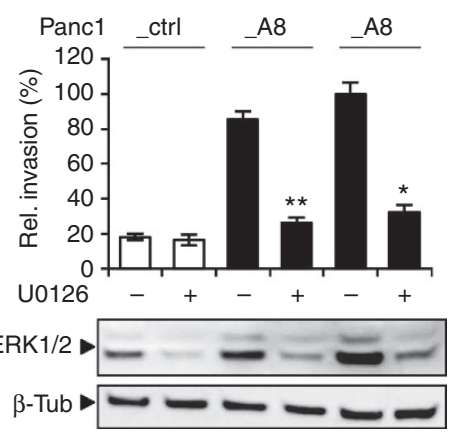

f
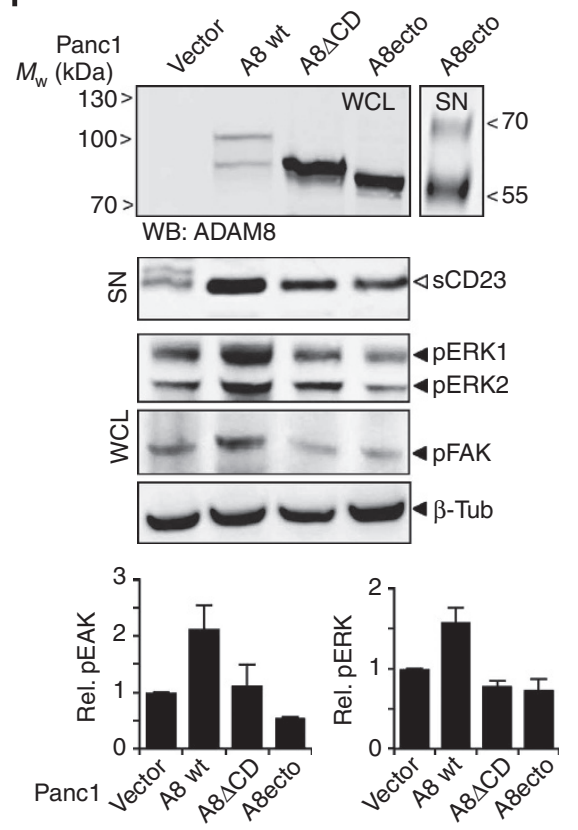

Figure 5 | ADAM8 intracellular kinase signalling. (a) Antibody detection of FAK phosphorylation (Y397) in Panc1_A8 and Panc1_ctrl cells $24 \mathrm{~h}$ after plating cells on plastic. Total FAK antibody was used to control equal loading and anti-ADAM8 confirms ADAM8 expression. A $\beta$-tubulin antibody was used as loading control. Note a 2.8-fold increase in pFAK in Panc1_A8 cells compared with Panc1_ctrl cells. (b) Western blotting (WB) of relative (rel.) phosphorylation of ERK1/2, in Panc1_ctrl and Panc1_A8 cells as determined by antibodies directed against phosphorylated forms of respective kinases. Lower panel, ERK1/2 phosphorylation in Panc1_ctrl and Panc1_A8 cells in the presence of $500 \mathrm{nM}$ BK-1361. (c) Relative invasiveness of Panc1_ctrl and Panc1_A8 cells (in duplicates) in the presence of ERK1/2 inhibitor U0126 (10 $\mu \mathrm{M})$ after $18 \mathrm{~h}$ incubation, indicating a strong correlation between ERK1/2 phosphorylation and invasion in Panc1 cells. ANOVA statistical test was used; ${ }^{\star} P<0.01,{ }^{\star \star} P<0.001$ (Student's $t$-test). (d) Correlation of ADAM8 and pERK1/2 levels in AsPC-1 control (shCtrl) and knockdown (shA8) cells. In clone AsPC-1_shA8/2, levels of pERK1/2 are reduced by threefold. (e) Relative kinase phosphorylation of the ERK and AKT signalling pathways after $18 \mathrm{~h}$ incubation with $(+)$ or without $(-)$ U0126 $(10 \mu \mathrm{M})$ in protein lysates of AsPC1_shCtrl and AsPC-1_shA8 cells. Kinases and their respective phosphorylation sites are indicated on the right. (f) Membrane-bound ADAM8 with cytoplasmic domain is required for FAK and ERK1/2 activation. Panc1 cells were transiently transfected with full-length ADAM8 construct (A8 wt), ADAM8 lacking the cytoplasmic domain (A8 $A C D$ ), or with a construct encoding the soluble ectodomain (A8ecto) of ADAM8, respectively. To control for cellular ADAM8 activity, the amount of soluble CD23 was determined in SNs by using an HA-tag antibody. The presence of ADAM8 protein variants, relative levels of $p F A K$ and $p E R K 1 / 2$ were detected by $W B$, using $\beta$-tubulin as loading control. Levels of FAK and ERK phosphorylation were quantified from three independent experiments, given as mean \pm s.e.m. $W C L$, whole-cell lysates.

The therapeutic effect of ADAM8 inhibition in vivo was analysed in mice with genotype Kras ${ }^{L S L-G 12 D}, \operatorname{Trp} 53^{R 172 H /+}$, $P d x^{C r e l}+(\text { KPC })^{2}$, a genetically engineered PDAC mouse model. Injections of BK-1361 were started around the onset of PanINs. KPC control groups received injections of either saline (as in a clinical setting) or CP (BK-1362). Control groups showed progression to PDAC with a median survival of 15.5 weeks for saline and 16 weeks for BK-1362. In contrast, BK-1361-treated KPC mice have extended median survival times of 24.2 weeks (Fig. 7a). The in vivo efficacy of BK-1361 was demonstrated by determining soluble ADAM8 levels (Fig. 7b). Lower frequencies of metastases in liver and lung were observed in BK-1361-treated KPC mice (Fig. 7c). Pancreas morphology in BK-1361-treated mice showed reduced infiltration areas in the pancreas compared with control mice (Fig. 7d,e), while the areas of intact acinar structures are increased $(0.74 \%$ for BK-1361-treated versus $0.18 \%$ for saline-treated mice, Fig. 7f). Tumour progression was associated with increased staining for ADAM8 and pERK1/2 in control KPC mice. In BK-1361-treated KPC mice, ADAM8 and $\mathrm{pERK} 1 / 2$ staining is restricted to acinar structures, suggesting that despite occurrence of neoplasias, tumour infiltration was reduced while the acinar architecture was more conserved 
a

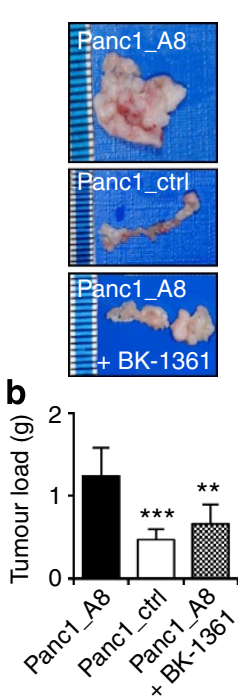

C
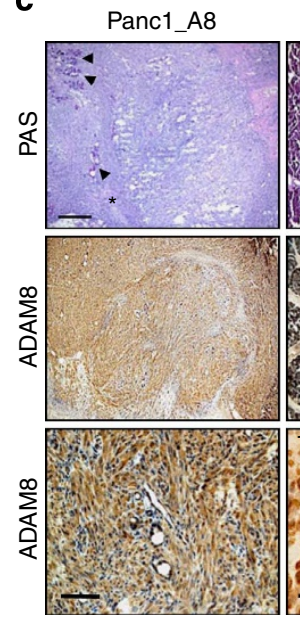

Panc1_ctrl

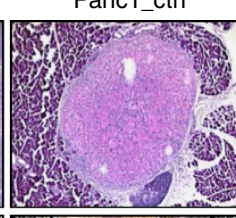

Panc1_A8 + BK-1361
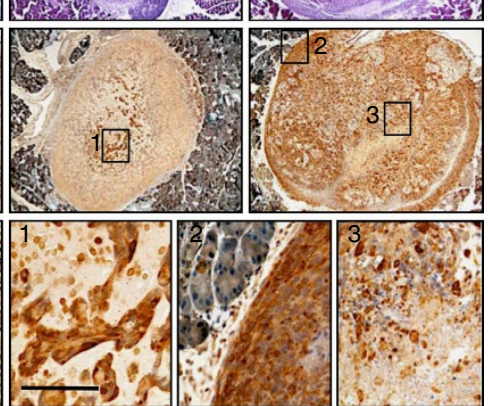

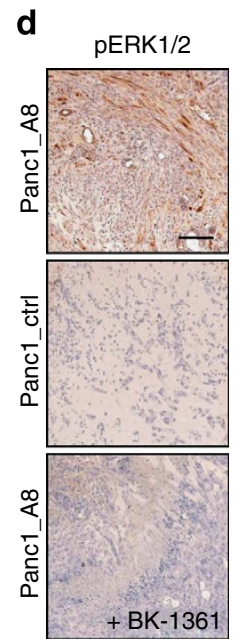

e

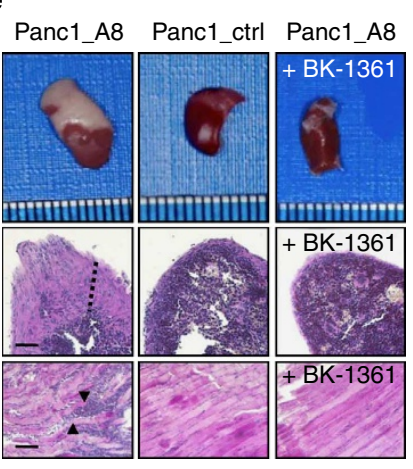

f

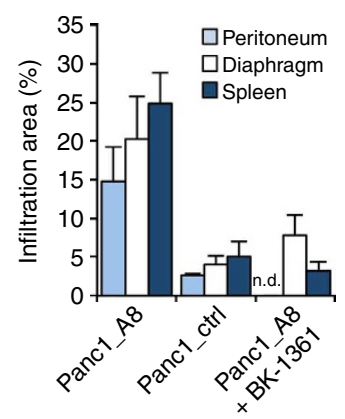

g

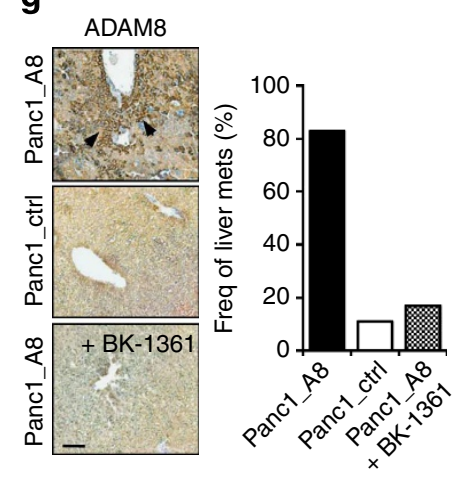

\section{$\mathbf{h}$}
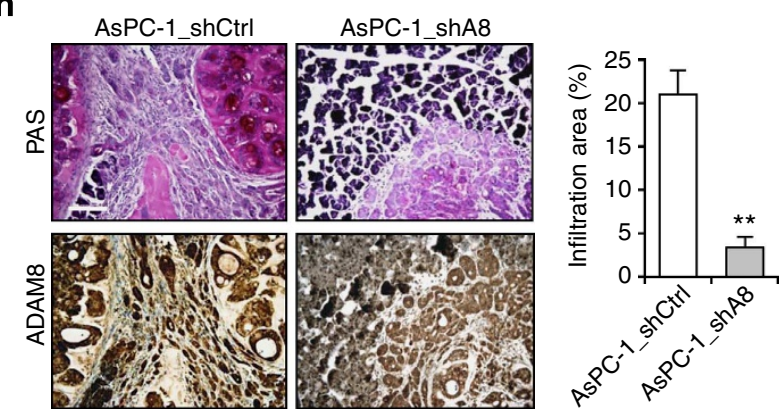

Figure 6 | Effect of ADAM8 inhibition in an orthotopic pancreatic cancer model. (a) Pancreas morphology after orthotopic injection of Panc1_A8, Panc1_ctrl or Panc1_A8 cells followed by BK-1361 injection ( $10 \mu \mathrm{g} \mathrm{g}^{-1}$ daily, $n=12$ per group). (b) Tumour load ( $\mathrm{g}$ ) 12 days after implantation ( $n=12$ ); Tukey's linear contrast test was used. ${ }^{\star \star} P<0.001,{ }^{\star \star \star} P<0.0001$ (Student's $t$-test) (c) Representative ADAM8 IHC/Periodic Acid Schiffs (PAS) staining of pancreas tumours from mice injected with Panc1_A8, Panc1_ctrl compared with implanted Panc1_A8/BK-1361 treated. Significant invasion of Panc1_A8 cells (arrows, upper panel left); across the implantation border (asterisk, upper left panel); Panc1_ctrl and Panc1_A8/BK-1361 injected tumours show less invasion from implantation site. Lower panel, ADAM8 staining; infiltration and loss of ductal architecture, increase in ADAM8 in Panc1_ctrl-implanted tumours and delineated between implantation site and surrounding pancreas tissue in Panc1_A8/BK-1361-treated mice. Lower left panel, enlarged image shows invasion of ADAM8-positive tumour cells; enlarged images (boxed in upper panel); box1: upregulation of ADAM8 in cell mass of Panc1_ctrl cells; box 2: enlarged view of the border between implantation site and surrounding ductal tissue; box 3: signs of intratumoral necrosis in tumours treated with BK-1361 (scale bars, $100 \mu \mathrm{m}$ ). (d) pERK1/2 staining of tumours. Only for Panc1_A8 (top), tumour cells stain positive for pERK1/2 (scale bar, $100 \mu \mathrm{m}$ ). (e) Morphology of spleen and diaphragm from mice injected with either Panc1_A8, Panc1_ctrl, or Panc1_A8/BK-1361. In PAS stains (lower panel), infiltration of tumour cells in the diaphragm is only seen in Panc1_A8. Scale bars, $100 \mu \mathrm{m}$. (f) Quantification of infiltration areas in peritoneum, diaphragm and spleen. Values are in \% of total tissue area \pm s.e.m. $(n=12)$. (g) ADAM8 ${ }^{+}$micrometastases in livers from Panc1_A8-injected mice; in contrast, metastases (mets) were mostly absent in livers from Panc1_ctrl and Panc1_A8/BK-1361-treated mice (scale bar, $200 \mu \mathrm{m}$ ); relative frequency (Freq.) of liver metastases in mice injected with Panc1_A8, Panc1_ctrl and Panc1_A8/BK-1361, respectively $(n=12)$. (h) Pancreas histology (PAS, ADAM8 IHC) after orthotopic implantation of AsPC-1_shCtrl and AsPC-1_shA8/2 cells. Scale bar, left upper panel, $100 \mu \mathrm{m}$. ADAM8 ${ }^{+}$cells in pancreas injected with AsPC-1_shCtrl cells, weaker staining with AsPC-1_shA8 cells. Infiltration areas in pancreata from six mice per group were quantified. Mean \pm s.e.m. ${ }^{\star \star} P<0.01$ (Student's $t$-test). 
(Fig. 7g). Staining intensities of pERK1/2 and ADAM8 is reduced in BK-1361-treated KPC mice (Fig. 7h), suggesting that, in vivo, ADAM8 inhibition leads to reduced activation of pERK1/2.

\section{Discussion}

Our study links the available clinical data on ADAM8 expression in pancreatic cancer cell lines and in $\mathrm{PDAC}^{15}$ to mechanistic data on ADAM8 in PDAC tumour progression. Using a novel proofof-concept ADAM8 inhibitor, we demonstrate that ADAM8 inhibition in PDAC leads to reduction of tumour load, infiltration and metastasis in vivo by affecting downstream signalling of ADAM8, thus further supporting the important role of ADAM8 in PDAC.

ERK1/2 signalling is considered a major pathway in PDAC, and the EGF/EGFR pathway has been established as an a

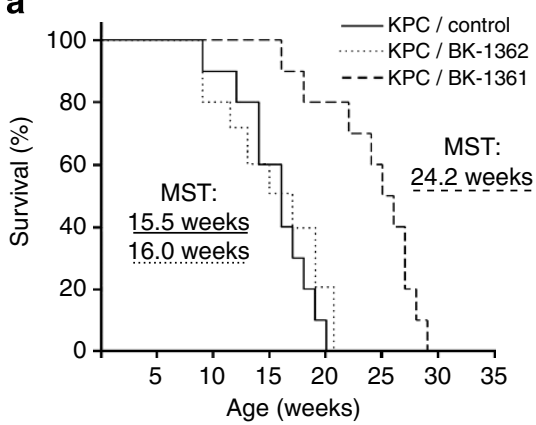

d

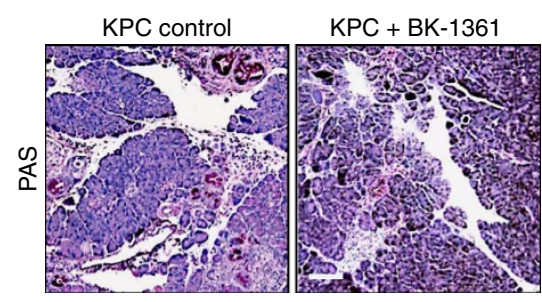

g

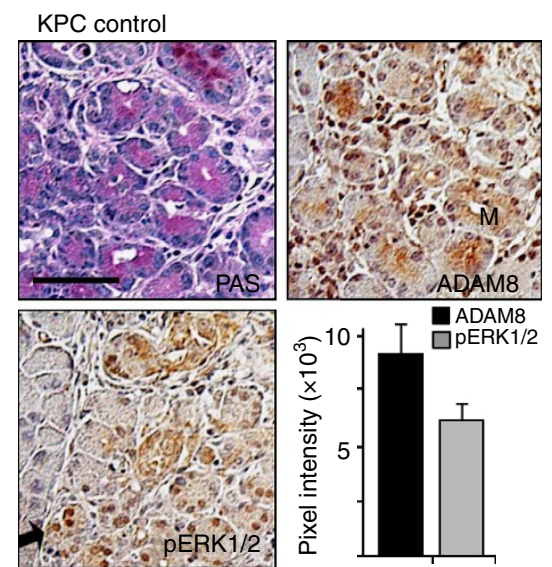

b
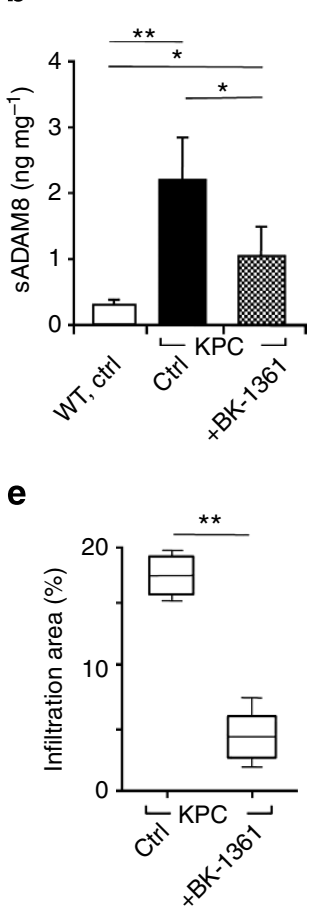

h

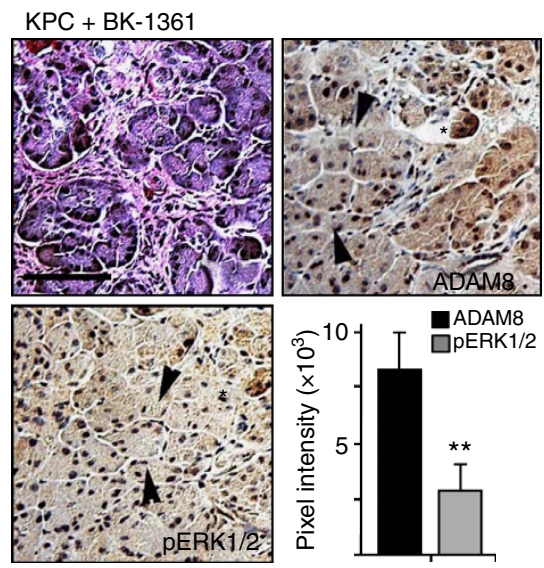

C

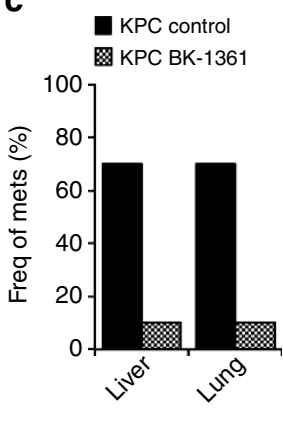

$\mathbf{f}$

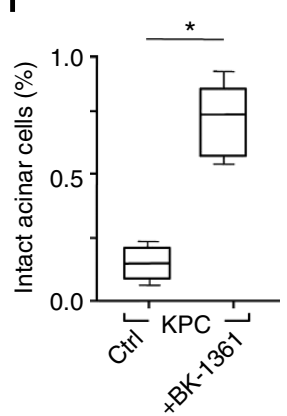

Figure 7 | Application of peptide BK-1361 in PDAC mice. (a) Kaplan-Meier analysis of KPC/saline, KPC/BK-1362 (CP) and KPC/BK-1361 mice $(n=10$ in each group). Median survival times (MSTs) are given in the diagram; statistical analysis was carried out using a log-rank test with $P<0.001$. (b) Concentration of soluble ADAM8 (sADAM8) in normal mouse pancreas (wt) and in pancreata of saline-treated and BK-1361-treated KPC mice, as determined by ELISA $(n=5)$ in $n g \mathrm{mg}^{-1}$ from pancreas. ANOVA was used as statistical test. ${ }^{\star} P<0.01,{ }^{\star \star} P<0.001$. (c) Frequency (Freq.) of metastases (mets) in liver and lung of KPC/control and KPC/BK-1361 mice. (d) IHC of mice at week 12. Representative Periodic Acid Schiffs (PAS) staining in KPC/saline versus KPC/BK-1361 pancreas; scale bar, $200 \mu \mathrm{m}$. (e) Whisker plot with quantification of invasive PDAC in saline and BK-1361-treated KPC mice. Tukey-type analysis was used for statistics; ${ }^{\star \star} P<0.001$ (Student's $t$-test). (f) Whisker plot of normal acinar area in KPC/saline and KPC/BK-1361treated pancreata $(n=5)$, Tukey-type analysis, ${ }^{\star} P<0.05$ (Student's $t$-test). (g,h) PAS staining, ADAM8 and pERK1/2 IHC in KPC/control $(\mathbf{g})$ and KPC/BK-1361 (h) mice. Arrowheads indicate focal acinar cell staining, arrow indicates nuclear staining and asterisks mark identical positions. Note loss of ductal architecture in control versus BK-1361-treated mice with positive staining for ADAM8 and pERK1/2. Quantification of IHC for KPC/saline and KPC/BK-1361 pancreas reveals less staining for pERK1/2 in BK-1361 versus saline-treated pancreata. Tukey-type statistical analysis was performed. ${ }^{\star *}<P<0.001$ (Student's $t$-test). Scale bar in $\mathbf{d}, 100 \mu \mathrm{m}$. 
upstream effector of ERK1/2, so that PDAC development and progression is associated with overexpression of EGFR ligands and ADAM17 (refs 43,44). Aberrant EGF signalling is associated with ERK1/2 signalling in tumour proliferation and migration of pancreatic cancer cells ${ }^{45}$. Since ADAM8 is an active shedding enzyme, evidence of a function of ADAM8 in EGF/EGFR signalling was investigated. No ADAM8-dependent shedding of EGFR ligands such as EGF, epiregulin, amphiregulin and HB-EGF was detected in substrate screens (Supplementary Fig. 8). This suggests that ADAM8 could act independent from the EGF/EGFR pathway, as demonstrated for ADAM10 and ADAM17 (ref. 14). Thus, it is likely that ADAM8 stimulates nonEGFR pathways for PDAC progression, which is in agreement with recent experimental observations that progression of PDAC is beyond sole EGF function ${ }^{46}$. For this hypothesis, we provide experimental evidence by demonstrating a link between ADAM8 and ERK1/2 signalling.

Our data suggest that ADAM8 interacts with $\beta 1$ integrin, an essential signalling module in $\mathrm{PDAC}^{47}$. The $\beta 1$-associated signalling pathways involving kinases such as FAK, p38, Akt and ERK $1 / 2$ are altered by expression of ADAM8. In particular, ERK1/2 activation is correlated with ADAM8 levels, as shown for cells either overexpressing ADAM8 or AsPC-1 cells bearing a genetic ADAM8 knockdown. In accordance with potent effects of ERK1/2 inhibitors PD98059 and U0126 on reducing Panc1 cell invasion, we propose an ADAM8-induced ERK1/2 activation via the ADAM8 cytoplasmic domain, thereby regulating MMP activities more effectively than inhibitors for Akt and p38.

Although ADAM8 can cleave fibronectin ${ }^{12}$, the effect of ADAM8 on cell migration into different ECM substrates could better be explained by ERK1/2 activation in Panc1 cells that regulates the extracellular activities of MMPs. In accordance, increased extracellular activities of MMP-2 and MMP-14 were detected by PrAMA assays. MMP-2 was shown to promote PDAC significantly ${ }^{48,49}$, and MMP-2 inhibitors were exploited in Panc1-derived xenografted tumours, resulting in reduced tumour $\operatorname{load}^{50}$. Moreover, the role of MMP-14 has been investigated in PDAC and its role in invasion into collagen $\mathrm{I}$, the most abundant ECM present in human PDAC, and its role in cancer invasion has been demonstrated ${ }^{8}$. MMP-2/ MMP-14 induction, however, was not detected on transcriptional levels (Supplementary Fig. 4). Thus, ADAM8-dependent kinase activation such as FAK and ERK1/2 may increase the release of MMP-2 and MMP-14, respectively. To support this notion, FAK and ERK1/2 were shown to regulate MMP release $\mathrm{s}^{51,52}$. MMP-9 is undetectable in Pancl cells, so that the effect of FAK and ERK1/2 on MMP release is restricted to MMP-2 and MMP-14, respectively. Their activities are interlinked as pro-MMP-2 activation and MMP-14 processing are concomitant events ${ }^{53}$.

Xenograft data demonstrate that ADAM8 causes increased invasiveness and tumour growth in vivo. In cell culture, serumdependent and -independent growth of Pancl cells was not affected, suggesting that the observed growth relates to infiltrative growth. It is interesting to note that in the orthotopic PDAC model, non-invasive Panc1 cells (Panc1_ctrl or Panc1_A8 cells in mice treated with BK-1361) showed intratumoral hypoxia and necrosis. Hypoxia stimulates ADAM8 expression in a range of PDAC cell lines and Pancl cells weakly in culture ${ }^{54}$; however, our data demonstrated a strong induction of ADAM8 under hypoxic conditions in vivo. It is likely that ADAM8 stimulates angiogenesis under hypoxic conditions, as shown earlier in a model of retinopathy ${ }^{34}$.

It is remarkable how significantly ADAM8 contributed to tumour cell invasion and metastasis in the PDAC models. Consistent with a proposed role of ADAM8 in PDAC progression, we performed a chemotherapy study in KPC mice by initiating BK-1361 treatment at the onset of PanINs $\sim 4$ weeks of age $^{2}$. Given the high degree of specificity reported for BK-1361 towards ADAM8 (see Table 2 for details), it is likely that BK-1361 has selectivity for ADAM8 over other ADAM proteases. However, since BK-1361 mimics an integrin-binding motif, offtarget effects may exist and could act on integrins, in particular on $\beta 1$ integrin. In this respect, unwanted side effects of BK-1361 by inhibition of non-tumour cell located in $\beta 1$ integrin should be excluded. However, our in vivo data in PDAC mice suggest that off-target effects by BK-1361 may not affect the therapeutic benefit, given the prolonged survival of KPC mice treated with BK-1361 (median survival 15.5 weeks for saline versus 24.2 weeks for BK-1361-treated KPC mice). In terms of percentage survival, ADAM8 inhibition in KPC mice was similarly effective as EGFR inhibition by erlotinib/gemcitabine combination therapy ${ }^{43}$, considering the stringency of the PDAC model used. For further clinical trials, a combination therapy might be beneficial with potentially additive effects of an ADAM8 inhibitory and an anti-EGFR therapy.

Our in vitro studies demonstrated that ADAM8 activity requires dimer or greater order multimerization. As reported for ADAM12 and ADAM17, complex formation revealed ambiguous results, similar to our observations of ADAM8 dimers and trimers that were detectable in native gels. However, the precise mechanism of higher order aggregate formation remains to be elucidated and a number of domains have been implied in this process ${ }^{55,56}$. From the literature, different aggregation mechanisms of domains functionally also present in ADAM8 have been proposed and there may be several modes of interaction $^{55,56}$. We hypothesize that these interactions may occur sequentially, as the ADAM8 DI domain interaction, blocked by BK-1361 prevents further complexes from forming. Since we detected dimers and trimers in the native gel at low concentrations of recombinant ADAM8, we interpret these data that dimers are formed by DI domain interactions, whereas trimers could be formed by different interactions involving the EGF-like domain, as a model for ADAM17 multimerization proposes ${ }^{55}$. Indeed, high concentrations of ADAM8 in vitro lead to higher order multimers such as aggregates of two dimers and/ or two trimers, thereby suggesting the presence of such interactions apart from the DI domain interactions (Supplementary Fig. 3e). Given the effect of BK-1361 on preventing any ADAM8-ADAM8 interactions, we conclude that initial dimer formation is a critical step in ADAM8 multimerization so that all higher aggregates are prevented from forming by BK-1361. For most ADAM family members, modelling of their DI domains revealed a so-called C-fold scaffold ${ }^{57}$ that is inaccessible at the position of the IBL, as shown in the X-ray structure for ADAM10 (ref. 41). Homology modelling of the ADAM8 DI domain based on ADAM10 structural data reveals that the IBL is significantly shifted towards an extended loop structure as this was shown for ADAM28 (ref. 58). Initial proof for the functionality of ADAM8binding peptides was obtained by blocking homophilic interactions in cell-binding assays ${ }^{36}$ with $\mathrm{EC}_{50}$ values in the low nanomolar range using BK-1361 and derived peptides. Since there is no homologous amino-acid sequence in the IBL of other ADAM proteases, our approach combines the advantage of high specificity with little or no off-target effects as judged by the lack of toxicity of BK-1361. In conjunction with no expected side effects of ADAM8 inhibition as deduced from ADAM8-deficient mice $^{28,29}$, ADAM8 inhibition might be an effective therapy option in mouse PDAC that accurately mirrors human pathology. As first-in-class inhibitor, BK-1361 is structurally similar to cilengitide, an $\alpha_{\mathrm{v}}$ integrin-binding angiogenesis inhibitor that raised no safety issues in patients, and is orally available but failed 
in phase III trials owing to unwanted side effects. Even a short in vivo half-life of $\sim 34$ min for BK-1361 (Supplementary Fig. 10), comparable to the half-life of cilengitide, should not limit the therapeutic use of BK-1361, and thereby provides a platform for further preclinical studies including alternative delivery routes and structure-activity relationship.

\section{Methods}

Mice. Kras ${ }^{\mathrm{LSL}}-\mathrm{G}^{2} \mathrm{D} ; \operatorname{Tr} p 53^{\mathrm{R} 172 \mathrm{H} /+} ; P d x^{\mathrm{Cre} /+}(\mathrm{KPC})$ were described earlier ${ }^{2}$. After weaning, all mice were genotyped. Equal numbers of male and female mice were taken for analysis. All animal experiments were conducted in accordance with Home Office regulations under a relevant project licence (T.H.) and with the German Law on the protection of animals, and were approved by the Local Government (Regierungspräsidium Giessen, J.W.B.).

Cell lines. Pancl and AsPC-1 cells were obtained from Sigma (UK); HEK-293, COS7 and MDA-MB-231 cells were purchased from American Type Culture Collection (ATCC). AsPC-1 cells were grown in RPMI medium; all other cell lines were grown in DMEM (Dulbecco's-modified Eagle medium, Invitrogen, Groningen, The Netherlands), with all media containing $10 \%$ fetal calf serum, $1 \%$ penicillin/streptomycin and $1 \%$ glutamine (Invitrogen, UK). For all experiments, cells were kept in humidified atmosphere at $37^{\circ} \mathrm{C} / 5 \% \mathrm{CO}_{2}$

Antibodies. Human recombinant ADAM8 ectodomain (1031-AD), recombinant ADAM9, ADAM10 and ADAM17, and MMP-2 and MMP-14, human phosphoMAPK Array Kit (ARY002B) and Proteome Profiler soluble receptor array (ARY012) were obtained from R\&D Systems (Abingdon, UK). For simultaneous detection of mouse and human ADAM8, a rabbit polyclonal antibody was purchased from Biorbyt (orb 4376, 1:1,000). For IP and native gel electrophoresis, an antibody against the ectodomain of ADAM8 (R\&D Systems, AF1031, 1:1,000) was used, for detection of human ADAM8 in western blots, we used an antibody against the cytoplasmic domain of ADAM8 (AB19017; Millipore, Watford, UK, 1:1,000). MMP-2 (R\&D Systems, 1;1,000), MMP-9 (Genetex, Irvine, USA, 1:2,000), MMP-14 (R\&D Systems, 1:1,000), Birch pollen profilin (BiPro 4A6, Antibody Facility, Braunschweig University, Germany, 1:2,000) and integrin $\beta 1$ (sc-8978; Santa Cruz Biotechnology, USA, 1:500). Detection of CD23 was performed using an anti-HA antibody (3F10, Mannheim, Germany, 1:1,000). Antibody 12G10 (Abcam, Cambridge, UK, 1:1,000) was used to detect activated integrin $\beta 1$. For blocking integrin $\beta 1$, antibody P4C10 from Millipore (1:500) was used. Specific antibodies against the phosphorylated forms of ERK1/2 (T202/Y204; Cell Signaling, Hitchin, UK, 1:1,000), Akt (Ser473; Cell Signaling, 1:1,000 and Thr308; Cell Signaling, 1:1,000), p38 $\gamma$ (T180/Y182; R\&D Systems, 1:1,000), Phophatase and tensin homolog (PTEN) (Ser380, Cell Signaling, 1:1,000), c-Raf (Ser 259, Cell Signaling, 1:1,000), MEK1/2 (Ser217/221, Cell Signaling, 1:1,000) and FAK (Y397; BD Biosciences, Oxford, UK, 1:1,000) were used. Horseradish peroxidase (HRP) conjugates antibodies were purchased from Sigma and Southern Biotech (both UK, 1:2,000) and fluorescently labelled antibodies were obtained from Invitrogen and Abcam (1:1,000). The inhibitor tablets cOmplete w/o EDTA and PhosSTOP were purchased from Roche.

Peptides. Cyclic peptides (named BK-n) were synthesized by Peptide 2.0 (Canada). Purities were $>97 \%$. Peptide identities were verified by high-performance liquid chromatography (HPLC) and mass spectrometry (MS).

Fluorescent peptides contain 5-carboxy-fluoresceine (FAM) as fluorophore and Dabcyl ([4-((4-(dimethylamino)phenyl)azo)benzoic acid]) as a quencher ${ }^{59}$ Dinitrophenyl-labelled peptides were purchased from the UNC Chapel Hill peptide synthesis laboratory.

Oligonucleotides. Oligonucleotides for PCR and site-directed mutagenesis were synthesized by Sigma.

Cloning of ADAM8 constructs. Full-length human ADAM8 cDNA was obtained by reverse transcription and PCR (hA8fw $5^{\prime}$-ATGCGCGGCCTCGGGCTCT- $3^{\prime}$, hA8Sto.as $5^{\prime}$-CTAGGGTGCTGTGGGAGCTCCG-3') from AsPC-1 cells. The PCR product was cloned into the expression vector pTarget (Promega) and the sequences of the ADAM8 constructs were verified by DNA sequencing. The C-terminally tagged ADAM8 constructs (EGFP, mCherry and BiPro ${ }^{36}$ ) were generated by PCR using cloning primers (hA8SgfI $5^{\prime}$-GAGGCGATCGCCATGC GCGGCCTCGGGCTC-3', hA8MluI 5'-GCGACGCGTGGGTGCTGTGGGAG CTCC) and ligated into the pCMV6 expression vector (Origene) using the Mlu and Sgfl restriction sites, respectively.

Transfection experiments. LTX Lipofectamine (Invitrogen) was used in cell transfection assays according to the manufacturer's instructions. Cells were lysed $48 \mathrm{~h}$ later in modified RIPA buffer (50 mM HEPES pH 7.4, $150 \mathrm{mM} \mathrm{NaCl}, 1 \%$ NP40, 0.5\% sodium deoxycholate, $0.1 \%$ SDS, $10 \mathrm{mM}$ 1,10-phenanthroline, cOmplete EDTA free and PhosSTOP) and sonicated. A Bradford assay (Thermo
Scientific) was used to determine the protein concentrations in whole-cell lysates and in SNs.

Generation of Panc1_A8 cells. Panc1 cells were co-transfected with a full-length ADAM8 construct (cloned in pTarget) and the pRFP-C-RS vector (Origene) encoding for red fluorescence protein (RFP). Control cells were transfected with RFP vector only. Twenty-four hours after transfection, cells were treated with the respective selection antibiotics $\left(1 \mu \mathrm{g} \mathrm{ml}^{-1}\right.$ puromycin and $\left.1 \mathrm{mg} \mathrm{ml}^{-1} \mathrm{G} 418\right)$. Resistant RFP-positive cells were isolated by fluorescence-activated cell sorting (FACS). ADAM8 expression in single-cell clones was analysed by western blotting and quantitative reverse transcription PCR.

Generation ADAM8 knockdown cells. AsPC-1 cells were stably transfected with $\mathrm{HuSH}$ shADAM8 constructs (TF314948, Origene) with the target sequence $5^{\prime}$-GCGGCACCTGCATGACAACGTACAGCTCA-3' and selected with puromycin $\left(1 \mu \mathrm{g} \mathrm{ml}^{-1}\right)$. Around 20 cell clones were selected and checked for successful knockdown of ADAM8. To generate control cell clones, a construct with a scramble shRNA sequence was used.

Quantitative PCR analysis. After reverse transcription using $1 \mathrm{mg}$ of total RNA quantitative PCR was performed using SYBR Green kits (Bioline, Luckenwalde, Germany) in a STEP-One Light cycler (ABI Systems, Weiterstadt, Germany). As a housekeeping control gene, acidic ribosomal gene XS13 was used. The primers were as follows: ADAM8: fw: $5^{\prime}$-ACAATGCAGAGTTCCAGATGC- $3^{\prime}$; rev: 5'-GGACCA CACGGAAGTTGAGTT-3'; XS13: 5' -TGGGCAAGAACACCATGATG-3'; rev: 5'-AGTTTCTCCAGAGCTGGGTTGT-3'.

ADAM8 ELISA. To determine soluble ADAM8 in mouse tissue, a commercial ADAM8 ELISA assay (Hoelzel Diagnostica, Cologne, Germany) was used according to the manufacturer's instructions. Pancreas tissues were homogenized in 10 volumes of ice-cold PBS containing $1 \mathrm{mM}$ ethylene glycol tetraacetic acid (EGTA) and cOmplete Inhibitor Mix (Roche), clarified by centrifugation (10,000 r.p.m., $10 \mathrm{~min}$ at $\left.4^{\circ} \mathrm{C}\right)$. SNs were used for ELISA.

In vitro autocatalysis assay with ADAM8. Inactive recombinant human ADAM8 (Met1-Pro497) was obtained from R\&D Systems. ADAM8 autocatalytic activation assays were performed in $50 \mathrm{mM}$ Tris, $10 \mathrm{mM} \mathrm{CaCl}_{2}$ and $150 \mathrm{mM} \mathrm{NaCl}$, $\mathrm{pH} 7.5$ (TCN) buffer for the indicated times at $37^{\circ} \mathrm{C}$. If applicable, peptides were diluted 1:1,000 in the assay. To avoid evaporation during longer incubation periods, the enzyme solutions were overlaid with mineral oil.

Native gel electrophoresis. To assess complex formation of recombinant pro-ADAM8, native gel electrophoresis was performed. Samples were dissolved in native buffer to retain protein complexes $(0.02 \% \beta$-glycerophosphate, $0.02 \%$ sodium orthovanadate, $0.04 \%$ EGTA, 0.6\% HEPES, 0.03\% EDTA, $0.58 \%$ sodium chloride, $1.5 \%$ dodecyl maltoside and $0.11 \%$ sodium pyrophosphate decahydrate; Abcam) with $100 \mathrm{ng}$ pro-ADAM8 in the presence of either $200 \mathrm{nM} \mathrm{CP}$ (BK-1362) or BK-1361 were prepared and run on a $4-16 \%$ native gel (Novex, Life Technologies, Darmstadt, Germany). As molecular weight marker, Novex Native Mark was used. Gels were blotted and stained with AF1031 (see antibody section)

Activity assays with ADAM8. Activity of recombinant ADAM8 catalytic/DI domain was monitored at 2 min intervals using the fluorescent substrate DabcylHGDQMAQKSK(5FAM)- $\mathrm{NH}_{2}$ (ref. 59), emulating the cleavage site of CD23, a physiological substrate for ADAM8 (Fourie et al. ${ }^{30}$ ). Assays were performed in a multiwell plate reader (Fluostar Optima, BMG Labtech, Offenburg, Germany) using an excitation wavelength of $485 \mathrm{~nm}$ and an emission of $530 \mathrm{~nm}$, with activity expressed as fluorescence units per hour. The substrate concentration was $10 \mu \mathrm{M}$ in assay buffer $\left(20 \mathrm{mM}\right.$ Tris, $\mathrm{pH} 8.0,10 \mathrm{mM} \mathrm{CaCl}_{2}$ and $6 \times 10^{-4} \%$ Brij-35). Reactions were run in a 96-well black-coated plate. The concentration of ADAM8 enzyme was typically $10 \mathrm{ng}$ per reaction. For inhibition assays using the cyclic peptide inhibitor, concentrations varied from $10 \mathrm{nM}$ up to $5 \mu \mathrm{M}$.

Determination of MMP/ADAM activities in PDAC cell lines. PrAMA analysis ${ }^{42}$ was performed as follows: Panc1, AsPC-1 cells and derivates were cultured in duplicate using a six-well plate for $24 \mathrm{~h}$ in serum-free DMEM medium without phenol red. Medium was removed, spun to remove cell fragments and set aside for assaying. Cells were washed with PBS and then scraped from the plate and resuspended in a $1.5-\mathrm{ml}$ tube in a cold solution of $0.25 \mathrm{M}$ sucrose, $50 \mathrm{mM}$ Tris, $\mathrm{pH}$ 8 , and a protease inhibitor mixture (cOmplete, Roche Applied Science). Cells were broken via pipetting up and down, and the suspension was spun at $13,000 \mathrm{~g}$ to pellet the membranes, which were resuspended and washed with sucrose buffer. After pelleting, membranes were resuspended in $200 \mu \mathrm{l}$ of sucrose buffer per well of cells. Protein concentrations were determined using the Bio-Rad BPA assay. The medium and membrane suspension were tested for MMP/ADAM activity by using the PrAMA analysis technique developed by Miller et al. ${ }^{41}$ using substrates 
PEPDab005, PEPDab010, PEPDab008, PEPDab013 and PEPDab014, which varied in their specificities towards different ADAM family members and MMPs. Briefly, $12.5 \mu \mathrm{M}$ substrate concentrations in $60 \mu \mathrm{l}$ of assay buffer (see above) were incubated with either $20 \mu \mathrm{l}$ of medium or $10 \mu \mathrm{l}$ of resuspended membranes. Fluorescence units versus time were monitored with a Fluostar BMG Optima using excitation and emission wavelengths of 485 and $530 \mathrm{nM}$, respectively. Specific protease activities were inferred with PrAMA by comparing the pattern of substrate cleavage rates for each sample to a matrix of known substrate specificities for ADAM8, ADAM10, ADAM17, MMP-2 and MMP-14 that were determined using purified enzymes (Miller et al. ${ }^{41}$ ). Before performing PrAMA, substrate cleavage rates were first converted to fold change over control measurements as indicated. Each PrAMA experiment was repeated three times and values for activities are given as relative to defined control measurements (a.u.).

Immunofluorescence. MDA-MB-231, Panc1 cells and stable derivatives were grown on coverslips, and were fixed with $3.7 \%$ paraformaldehyde. ADAM8 was detected in unpermeabilized cells by goat polyclonal antibody AF1031 (R\&D Systems) as primary antibody. As secondary antibody, we used goat-anti-rabbitCy3 (1:500, Sigma). Images were acquired by confocal microscopy imaging using a Nikon A1R microscope equipped with CFI Plan Fluor $\times 40$ oil objective. Images were captured and exported with NIS Elements software (Nikon) and presented as TIF files.

Immunoblotting and IP experiment. Equal amounts of protein were loaded onto $10 \%$ reducing polyacrylamide (PAA) gels. The proteins were transferred on nitrocellulose, unspecific binding sites were blocked (5\% skimmed milk, $0.1 \%$ Tween-20 in PBS) and specific antibodies were used to detect the proteins of interest. An enhanced chemiluminescent (ECL) substrate (Thermo Scientific) for HRP enzyme and a chemiluminescence reader (Intas, Goettingen, Germany) were used for visualization. Images in Figs 4 and 5 have been cropped for presentation. Full size images are presented in Supplementary Figs 11 and 12.

For IP assays, the cells were lysed in co-IP buffer (50 mM HEPES pH 7.4, $150 \mathrm{mM} \mathrm{NaCl}_{2}, 1 \% \mathrm{NP} 40,0,5 \%$ sodium deoxycholate, $1,5 \mathrm{mM} \mathrm{MgCl}_{2}$ and complete). The sonicated lysates were centrifuged two times at full speed to remove cell debris. Cleared lysates were subjected to IP using specific antibodies in concentrations of $0.2 \mu \mathrm{g} \mathrm{ml}^{-1}$ (for anti-BiPro) and $2.5 \mathrm{\mu g} \mathrm{ml}^{-1}$ (for anti-ADAM8) overnight at $4{ }^{\circ} \mathrm{C}$. As unspecific binding controls, IgG was used instead of specific antibodies. After binding of precipitated material to Protein-G-sepharose and washing steps, the precipitated proteins were eluted by boiling in $2 \times$ SDS-loading buffer and were analysed by western blotting.

Gelatine zymography. Protein samples were prepared in non-reducing sample buffer without boiling before the experiment. Gelatin $(0.1 \%)$ was added in a separating gel to co-polymerize with PAA. During electrophoresis, proteins are separated in the PAA gel, while SDS present in the gel preserves MMPs in an inactive state. After the run, gels were washed with renaturing buffer $(2.5 \%$ Triton $\mathrm{X}-100 ; 2 \times 30 \mathrm{~min}$ each) that resulted in partially renatured MMPs with restored activity. The gel was incubated in developing buffer $(50 \mathrm{mM}$ Tris, $\mathrm{pH} 7.5,200 \mathrm{mM}$ $\mathrm{NaCl}, 4 \mathrm{mM} \mathrm{CaCl}$ and $0.02 \%$ Brij- $35 ; 30 \mathrm{~min}$ at RT, followed by $24 \mathrm{~h}$ at $37^{\circ} \mathrm{C}$ in fresh developing buffer). Next day, the gel was dyed in Coomassie staining buffer for $1 \mathrm{~h}$ followed by Coomassie destaining solution to visualize bands of active enzyme.

FRET/FLIM. FLIM was performed and data were analysed as described previously ${ }^{60}$. FLIM capability was provided by time-correlated single-photon counting electronics (Becker \& Hickl, SPC 700). Widefield acceptor monomeric Red Fluorescent protein (mRFP) images were acquired using a CCD camera (Hamamatsu) at exposure times of $<100 \mathrm{~ms}$. Data were analysed using TRI2 software (developed by Dr Paul Barber). All histogram data are plotted as mean FRET efficiency from $>12$ cells per sample. Lifetime images of exemplary cells are presented using a pseudocolour scale whereby blue depicts normal GFP lifetime (that is, no FRET) and red depicts reduced GFP lifetime (areas of FRET). Each experiment was repeated at least three times. Analysis of variance (ANOVA) was used to test statistical significance between different populations of data.

Activated MAPK assay. The human phospho-MAPK Array Kit (ARY002B; R\&D Systems) was used to analyse the influence of ADAM8 on the activation state of altogether 26 MAPKs and other serine/threonine kinases. The assay was used according to the manufacturer's instructions. In brief, cells of the stable control PANC1 clone and the ADAM8 overexpressing clone were lysed in lysis buffer 6, sonicated and a Bradford assay was used to determine the protein concentrations. Each array membrane was incubated in $2 \mathrm{ml}$ array buffer 5 for $1 \mathrm{~h}$ at room temperature on a rocker. An amount of $1 \mathrm{mg}$ of each lysate was mixed with array buffer 1 to a final volume of $1.5 \mathrm{ml}$. A volume of $20 \mu \mathrm{l}$ reconstituted Detection Antibody Cocktail was added and the mixture was incubated for $1 \mathrm{~h}$ at room temperature. After removal of array buffer 5 from the membranes, the lysate Detection Antibody mixture was added to the membrane. The membranes were incubated at $4{ }^{\circ} \mathrm{C}$ on a rocker overnight. Each membrane was washed three times with $1 \times$ wash buffer $(20 \mathrm{ml}, 10 \mathrm{~min}$ each) and incubated with $2 \mathrm{ml}$ streptavidin-HRP (1:2,000 in array buffer 5) for $30 \mathrm{~min}$ at room temperature on a rocker. After three repeating washing steps with $1 \times$ wash buffer $(20 \mathrm{ml}, 10 \mathrm{~min}$ each), an ECL substrate (Thermo Scientific) for HRP enzyme and an ECL camera (Intas, Goettingen, Germany) were used to detect the signals. The signals were quantified with the National Institutes of Health (NIH) ImageJ software package (freeware).

ADAM8 DI structure modelling. A BLAST (a) search of the Protein Data Bank (b) using the ADAM8 sequence revealed the top structure hits as Russell's viper venom metalloproteinase $(2 \mathrm{e} 3 \mathrm{x})(\mathrm{c})$ and vascular apoptosis-inducing protein-2 (2ero) (d). 2E3X and 2ERO align structurally at $2.33 \AA$ using the alignment in Supplementary Fig. 1. The structure of ADAM10 (2AO7) (e) was also aligned even though it was eighteenth in the BLAST results. The per cent identity matrix is shown in Supplementary Fig. 2.

Administration of cyclic peptide BK-1361 in mice. Lyophilized cyclic peptide was dissolved in sterile PBS to a concentration of $1 \mu \mathrm{g} \mu \mathrm{l}^{-1}$. Since neither an acute (single injection) nor a chronic toxicity (repeated weekly injections over a total of 4 weeks) using a dosage of $10 \mathrm{\mu gg}^{-1}$ body weight via intraperitoneal route was observed based on organ histology after necropsy (Supplementary Fig. 9), this dosage regimen was administered throughout the experiments presented in this study. Equal volumes of sterile PBS were injected as control. For KPC mice, BK1361 injections were started at 4 weeks of age and continued for over 8 weeks. Mice were constantly monitored and were killed when they reached end point criteria.

Orthotopic injections. Orthotopic pancreatic cancer tumours were implanted into 6-week-old female CD1 nu/nu mice. Ketamine/xylazine $\left(100 / 10 \mathrm{mg} \mathrm{kg}^{-1}\right)$ was used for anaesthesia. The left side of the mouse was shaved and the fur was completely removed. The surgical area was sterilized with an iodine solution (Povidone-iodine, Novaplus, Irving, TX, USA) and a small incision $(<1 \mathrm{~cm})$ was made through the skin and abdominal wall at the base of the spleen. The spleen was gently pulled through the incision, exposing the tail of the pancreas. The cellMatrigel solution $\left(1 \times 10^{6}\right.$ cells in $\left.50 \mu \mathrm{l}\right)$ was injected into the tail of the pancreas. The Matrigel was allowed to set $(\sim 10 \mathrm{~s})$, the needle was gently removed from the pancreas and the area was swabbed with iodine to devitalize any stray cells in the injection site. The pancreas and spleen were replaced in the abdomen and the incision site closed with 3-4 sutures (Ethilon 5-0 PS-3, Ethicon, Piscataway, NJ, USA). The Matrigel method of orthotopic tumour implantation has resulted in $100 \%$ tumour uptake with little evidence of extra-pancreatic leakage. Pancl and AsPC-1 cell-derived tumours were analysed 12 days after implantation.

Statistical analysis. Invasion assays and western blot data were analysed by oneway ANOVA. For in vivo experiments, two-way ANOVA using Shapiro-Wilk normality was used. For pairwise comparisons of tumour weights/volumes and tissue pathologies (acinar tissue and infiltration rates), including ImageJ analyses, Tukey-type linear contrast tests were used. Survival was estimated as a KaplanMeier survival curve, and the statistical analysis was carried out using a log-rank test for the censored data. Based on the obtained results, the data were considered not significant $(P \geq 0.05)$, significant ${ }^{*}(P \leq 0.05)$, highly significant ${ }^{* *}(P \leq 0.01)$ or very highly significant ${ }^{* * *}(P \leq 0.001)$.

\section{References}

1. Erkan, M. et al. The role of stroma in pancreatic cancer: diagnostic and therapeutic implications. Nat. Rev. Gastroenterol. Hepatol. 9, 454-467 (2012).

2. Hingorani, S. R. et al. Preinvasive and invasive ductal pancreatic cancer and its early detection in the mouse. Cancer Cell 4, 437-450 (2003).

3. Hezel, A. F., Kimmelman, A. C., Stanger, B. Z., Bardeesy, N. \& Depinho, R. A Genetics and biology of pancreatic ductal adenocarcinoma. Genes Dev. 20, 1218-1249 (2006).

4. Tuveson, D. A. \& Neoptolemos, J. P. Understanding metastasis in pancreatic cancer: a call for new clinical approaches. Cell 148, 21-23 (2012)

5. Haeno, H. et al. Computational modeling of pancreatic cancer reveals kinetics of metastasis suggesting optimum treatment strategies. Cell 148, 362-375 (2012).

6. Rhim, A. D. et al. EMT and dissemination precede pancreatic tumor formation Cell 148, 349-361 (2012).

7. Kong, X., Li, L., Li, Z. \& Xie, K. Targeted destruction of the orchestration of the pancreatic stroma and tumor cells in pancreatic cancer cases: molecular basis for therapeutic implications. Cytokine Growth Factor Rev. 23, 343-356 (2012).

8. Shields, M. A., Dangi-Garimella, S., Redig, A. J. \& Munshi, H. G. Biochemical role of the collagen-rich tumour microenvironment in pancreatic cancer progression. Biochem. J. 441, 541-552 (2012).

9. Murphy, G. The ADAMs: signalling scissors in the tumour microenvironment Nat. Rev. Cancer 8, 929-941 (2008).

10. Hanahan, D. \& Coussens, L. M. Accessories to the crime: functions of cells recruited to the tumor microenvironment. Cancer Cell 21, 309-322 (2012).

11. Mochizuki, S. \& Okada, Y. ADAMs in cancer cell proliferation and progression. Cancer Sci. 98, 621-628 (2007).

12. Zack, M. D. et al. ADAM-8 isolated from human osteoarthritic chondrocytes cleaves fibronectin at Ala(271). Arthritis Rheum. 60, 2704-2713 (2009). 
13. Moss, M. L. \& Bartsch, J. W. Therapeutic benefits from targeting of ADAM family members. Biochemistry 43, 7227-7235 (2004).

14. Blobel, C. P. ADAMs: key components in EGFR signalling and development. Nat. Rev. Mol. Cell Biol. 6, 32-43 (2005).

15. Valkovskaya, N. et al. ADAM8 expression is associated with increased invasiveness and reduced patient survival in pancreatic cancer. J. Cell. Mol. Med. 11, 1162-1174 (2007).

16. Yoshida, S., Setoguchi, M., Higuchi, Y., Akizuki, S. \& Yamamoto, S. Molecular cloning of cDNA encoding MS2 antigen, a novel cell surface antigen strongly expressed in murine monocytic lineage. Int. Immunol. 2, 585-591 (1990).

17. Yoshiyama, K., Higuchi, Y., Kataoka, M., Matsuura, K. \& Yamamoto, S. CD156 (human ADAM8): expression, primary amino acid sequence, and gene location. Genomics 41, 56-62 (1997).

18. Schlomann, U., Rathke-Hartlieb, S., Yamamoto, S., Jockusch, H. \& Bartsch, J. W. Tumor necrosis factor alpha induces a metalloproteasedisintegrin, ADAM8 (CD 156): implications for neuron-glia interactions during neurodegeneration. J. Neurosci. 20, 7964-7971 (2000).

19. Koller, G. et al. ADAM8/MS2/CD156, an emerging drug target in the treatment of inflammatory and invasive pathologies. Curr. Pharm. Des. 15, 2272-2281 (2009).

20. Wildeboer, D., Naus, S., Amy Sang, Q. X., Bartsch, J. W. \& Pagenstecher, A. Metalloproteinase disintegrins ADAM8 and ADAM19 are highly regulated in human primary brain tumors and their expression levels and activities are associated with invasiveness. J. Neuropathol. Exp. Neurol. 65, 516-527 (2006).

21. Ishikawa, N. et al. ADAM8 as a novel serological and histochemical marker for lung cancer. Clin. Cancer Res. 10, 8363-8370 (2004).

22. Fritzsche, F. R. et al. ADAM8 expression in prostate cancer is associated with parameters of unfavorable prognosis. Virchows Arch. 449, 628-636 (2006).

23. Zielinski, V. et al. ADAM8 in squamous cell carcinoma of the head and neck: a retrospective study. BMC Cancer 12, 76 (2012).

24. Zhang, R., Yuan, Y., Zuo, J. \& Liu, W. Prognostic and clinical implication of a disintegrin and metalloprotease 8 expression in pediatric medulloblastoma. J. Neurol. Sci. 323, 46-51 (2012).

25. Li, Z. et al. Upregulation of a disintegrin and metalloprotease 8 influences tumor metastasis and prognosis in patients with osteosarcoma. Pathol. Oncol. Res. 18, 657-661 (2012).

26. Romagnoli, M. et al. ADAM8 expression in invasive breast cancer promotes tumor dissemination and metastasis. EMBO Mol. Med. 6, 278-294 (2014).

27. Kelly, K. et al. Metalloprotease-disintegrin ADAM8: expression analysis and targeted deletion in mice. Dev. Dyn. 232, 221-231 (2005).

28. Bartsch, J. W. et al. Tumor necrosis factor-alpha (TNF-alpha) regulates shedding of TNF-alpha receptor 1 by the metalloprotease-disintegrin ADAM8: evidence for a protease-regulated feedback loop in neuroprotection. J. Neurosci. 30, 12210-12218 (2010).

29. Gómez-Gaviro, M. et al. Expression and regulation of the metalloproteinase ADAM-8 during human neutrophil pathophysiological activation and its catalytic activity on L-selectin shedding. J. Immunol. 178, 8053-8063 (2007).

30. Fourie, A. M., Coles, F., Moreno, V. \& Karlsson, L. Catalytic activity of ADAM8, ADAM15, and MDC-L (ADAM28) on synthetic peptide substrates and in ectodomain cleavage of CD23. J. Biol. Chem. 278, 30469-30477 (2003).

31. Naus, S. et al. Identification of candidate substrates for ectodomain shedding by the metalloprotease-disintegrin ADAM8. Biol. Chem. 387, 337-346 (2006).

32. Naus, S. et al. Ectodomain shedding of the neural recognition molecule CHL1 by the metalloprotease-disintegrin ADAM8 promotes neurite outgrowth and suppresses neuronal cell death. J. Biol. Chem. 279, 16083-16090 (2004).

33. Guaiquil, V. H. et al. ADAM8 is a negative regulator of retinal neovascularization and of the growth of heterotopically injected tumor cells in mice. J. Mol. Med. (Berl) 88, 497-505 (2010).

34. Hernández, I., Moreno, J. L., Zandueta, C., Montuenga, L. \& Lecanda, F. Novel alternatively spliced ADAM8 isoforms contribute to the aggressive bone metastatic phenotype of lung cancer. Oncogene 29, 3758-3769 (2010).

35. Schlomann, U. et al. The metalloprotease disintegrin ADAM8. Processing by autocatalysis is required for proteolytic activity and cell adhesion. J. Biol. Chem. 277, 48210-48219 (2002).

36. Eto, K. et al. Functional classification of ADAMs based on a conserved motif for binding to integrin alpha 9beta 1: implications for sperm-egg binding and other cell interactions. J. Biol. Chem. 277, 17804-17810 (2002).

37. Zhang, X. P., Kamata, T., Yokoyama, K., Puzon-McLaughlin, W. \& Takada, Y. Specific interaction of the recombinant disintegrin-like domain of MDC-15 (metargidin, ADAM-15) with integrin alphavbeta3. J. Biol. Chem. 273, 7345-7350 (1998)

38. Zigrino, P. et al. Role of ADAM-9 disintegrin-cysteine-rich domains in human keratinocyte migration. J. Biol. Chem. 282, 30785-30793 (2007).

39. Rao, H. et al. Alpha9beta1: a novel osteoclast integrin that regulates osteoclast formation and function. J. Bone Miner. Res. 21, 1657-1665 (2006).
40. Janes, P. W. et al. Adam meets Eph: an ADAM substrate recognition module acts as a molecular switch for ephrin cleavage in trans. Cell 123, 291-304 (2005).

41. Miller, M. A. et al. Proteolytic Activity Matrix Analysis (PrAMA) for simultaneous determination of multiple protease activities. Integr. Biol. (Camb) 3, 422-438 (2011).

42. Lewis, J. M. \& Schwartz, M. A. Mapping in vivo associations of cytoplasmic proteins with integrin beta 1 cytoplasmic domain mutants. Mol. Biol. Cell. 6, 151-160 (1995).

43. Ardito, C. M. et al. EGF receptor is required for KRAS-induced pancreatic tumorigenesis. Cancer Cell 22, 304-317 (2012).

44. Navas, C. et al. EGF receptor signalling is essential for k-ras oncogene-driven pancreatic ductal adenocarcinoma. Cancer Cell 22, 318-330 (2012).

45. Botta, G. P., Reginato, M. J., Reichert, M., Rustgi, A. K. \& Lelkes, P. I. Constitutive K-RasG12D activation of ERK2 specifically regulates 3D invasion of human pancreatic cancer cells via MMP-1. Mol. Cancer Res. 10, 183-196 (2012).

46. Seton-Rogers, S. Tumorigenesis: pushing pancreatic cancer to take off. Nat. Rev Cancer 12, 739 (2012).

47. Grzesiak, J. J. et al. Knockdown of the $\beta(1)$ integrin subunit reduces primary tumor growth and inhibits pancreatic cancer metastasis. Int. J. Cancer 129, 2905-2915 (2011).

48. Layton, T., Stalens, C., Gunderson, F., Goodison, S. \& Silletti, S. Syk tyrosine kinase acts as a pancreatic adenocarcinoma tumor suppressor by regulating cellular growth and invasion. Am. J. Pathol. 175, 2625-2636 (2009).

49. Grippo, P. J. et al. Concurrent PEDF deficiency and Kras mutation induce invasive pancreatic cancer and adipose-rich stroma in mice. Gut 61, 1454-1464 (2012).

50. Lu, G. et al. Selection of peptide inhibitor to matrix metalloproteinase-2 using phage display and its effects on pancreatic cancer cell lines PANC-1 and CFPAC-1. Int. J. Biol. Sci. 8, 650-662 (2012).

51. Segarra, M. et al. Dual function of focal adhesion kinase in regulating integrininduced MMP-2 and MMP-9 release by human T lymphoid cells. FASEB J. 19, 1875-1877 (2005).

52. Liu, J. et al. BMP2 induces PANC-1 cell invasion by MMP-2 overexpression through ROS and ERK. Front. Biosci. (Landmark Ed) 17, 2541-2549 (2012).

53. Stanton, H. et al. The activation of ProMMP-2 (gelatinase A) by HT1080 fibrosarcoma cells is promoted by culture on a fibronectin substrate and is concomitant with an increase in processing of MT1-MMP (MMP-14) to a 45 $\mathrm{kDa}$ form. J. Cell. Sci. 111, 2789-2798 (1998).

54. Valkovskaya, N. V. Hypoxia-dependent expression of ADAM8 in human pancreatic cancer cell lines. Exp. Oncol. 30, 129-132 (2008).

55. Lorenzen, M., Trad, A. \& Grötzinger, J. Multimerisation of A disintegrin and metalloprotease protein-17 (ADAM17) is mediated by its EGF-like domain. Biochem. Biophys. Res. Commun. 415, 330-336 (2011).

56. Stautz, D. et al. Cell-surface metalloprotease ADAM12 is Internalized by a clathrin- and Grb2-dependent mechanism. Traffic 13, 1532-1546 (2012).

57. Takeda, S., Igarashi, T., Mori, H. \& Araki, S. Crystal structures of VAP1 reveal ADAMs' MDC domain architecture and its unique C-shaped scaffold. EMBO J. 25, 2388-2396 (2006).

58. Bridges, L. C., Hanson, K. R., Tani, P. H., Mather, T. \& Bowditch, R. D. Integrin alpha4betal-dependent adhesion to ADAM 28 (MDC-L) requires an extended surface of the disintegrin domain. Biochemistry 42, 3734-3741 (2003).

59. Moss, M. L. \& Rasmussen, F. H. Fluorescent substrates for the proteinases ADAM17, ADAM10, ADAM8, and ADAM12 useful for high-throughput inhibitor screening. Anal. Biochem. 366, 144-148 (2007).

60. Parsons, M., Messent, A. J., Humphries, J. D., Deakin, N. O. \& Humphries, M. J. Quantification of integrin receptor agonism by fluorescence lifetime imaging. J. Cell. Sci. 121, 265-271 (2008).

\section{Acknowledgements}

J.W.B. was supported by Cancer Research Technology (CRT) and the Adolf-Schmidtmann Foundation Marburg to C.C., by Cancer Research UK (C18270/A12888 and C18270/A14355) to T.H., Pancreatic Cancer Research Fund (T.H.). The research leading to these results has received funding from the People Programme (Marie Curie Actions) of the European Union's Seventh Framework Programme FP7/2007-2013 under REA grant agreement no. 317445; we acknowledge Drs L. Fletcher, I. Patzak and J. Little (CRT) for their continuous support and many helpful discussions, S. Motzny for expert technical assistance, W. Schulz for immunohistochemistry, S. Kramer for ADAM8- $\Delta$ CD construct and Professor A. Pagenstecher/Dr M. Hofer (Marburg) for help with in vivo analyses. We furthermore acknowledge Zena Werb for the kind gift of a CD23-HA expression construct and Dr K. Bruce and Dr S. Naus for critical reading of the manuscript.

\section{Author contributions}

This study was conceived by J.W.B., D.A.T., C.N., D.A.L., T.H., M.P., M.L.M. and N.S.; experiments were designed by J.W.B., G.K., U.S. and M.L.M.; acquisition of all the data 
was done by U.S., G.K., C.C., T.F., P.G., A.M.G., S.H., P.C., R.S., M.B., R.R., R.R.K., F.H.R. and M.A.M.; the analysis and interpretation of data was performed by U.S., G.K., C.C., J.W.B., M.L.M., D.A.L. and M.A.M.; the final manuscript was prepared by U.S., G.K., C.C., M.L.M. and J.W.B.; and the whole study was supervised by J.W.B.

\section{Additional information}

Supplementary Information accompanies this paper at http://www.nature.com/ naturecommunications
Competing financial interests: The authors declare no competing financial interests.

Reprints and permission information is available online at http://npg.nature.com/ reprintsandpermissions/

How to cite this article: Schlomann, U. et al. ADAM8 as a drug target in pancreatic cancer. Nat. Commun. 6:6175 doi: 10.1038/ncomms7175 (2015). 